\title{
Sulfur-Containing, Quinodimethane Embedded Acene Analogue with Nine Consecutively Fused Six-Membered Rings
}

Yang Chen, Huilin Kueh, Tullimilli Y. Gopalakrishna, Shaoqiang Dong, Yi Han and Chunyan Chi*

Department of Chemistry, National University of Singapore, 3 Science Drive 3, 117543, Singapore

Fax: (+65) 6779 1691, E-mail: chmcc@nus.edu.sg

\section{Contens}

1. Experimental Section $\mathrm{S} 2$

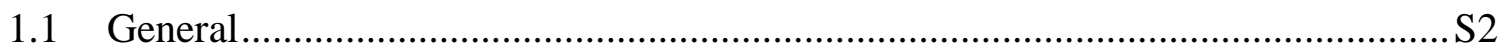

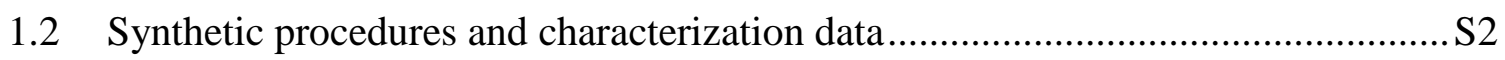

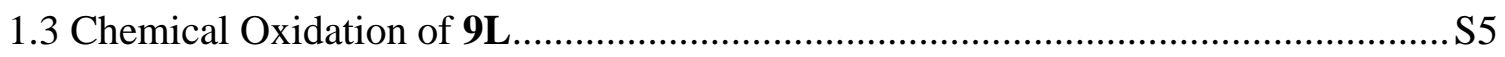

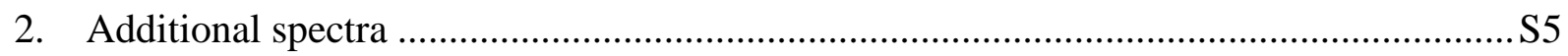

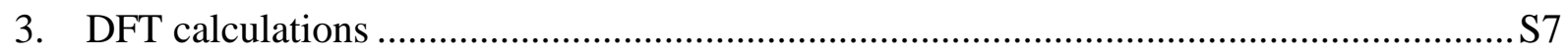

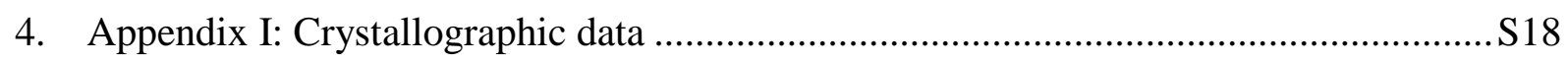

5. Appendix II: ${ }^{1} \mathrm{H}$ NMR and ${ }^{13} \mathrm{C}$ NMR spectra of all new compounds ......................... S20

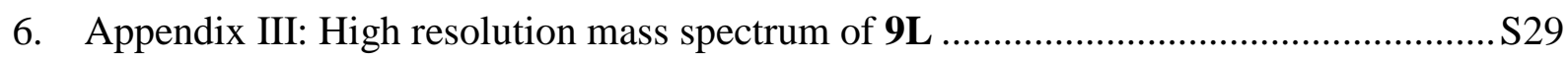




\section{Experimental Section}

\subsection{General}

All reagents were purchased from commercial sources without further purification. Anhydrous dichloromethane (DCM) and dimethylformamide (DMF) was distilled from $\mathrm{CaH}_{2}$. Anhydrous toluene and THF were distilled from sodium-benzophenone immediately prior to use. ${ }^{1} \mathrm{H}$ and ${ }^{13} \mathrm{C}$ NMR spectra were recorded using Advance $500 \mathrm{MHz}$ Bruker spectrometer in $\mathrm{CDCl}_{3}$, $\mathrm{CD}_{2} \mathrm{Cl}_{2}$ or $\mathrm{C}_{6} \mathrm{D}_{6}$ with tetramethylsilane (TMS) as the internal standard. The chemical shift was recorded in ppm and the following abbreviations were used to explain the multiplicities: $\mathrm{s}=$ singlet, $\mathrm{d}=$ doublet, $\mathrm{t}=$ triplet, $\mathrm{m}=$ multiplet, $\mathrm{br}=$ broad. High-resolution $(\mathrm{HR})$ APCI mass spectra were recorded on a MicrOTOF-QII instrument. UV-vis absorption spectra were recorded on a Shimadzu UV-3600 spectrophotometer. Cyclic voltammetry and differential pulse voltammetry measurements were performed in dry dichloromethane on a CHI 620C electrochemical analyzer with a three-electrode cell, using $0.1 \mathrm{M} \mathrm{Bu}_{4} \mathrm{NPF}_{6}$ as supporting electrolyte, $\mathrm{AgCl} / \mathrm{Ag}$ as reference electrode, gold disk as working electrode, $\mathrm{Pt}$ wire as counter electrode, and scan rate at $50 \mathrm{mV} \mathrm{s}^{-1}$. The potential was externally calibrated against the ferrocene/ferrocenium couple. Single crystals were measured at low temperature ( $T=100 \mathrm{~K}$ ) on a four circles goniometer Kappa geometry Bruker AXS D8 Venture equipped with a Photon 100 CMOS active pixel sensor detector using a Molybdenum monochromatized $(\lambda=0.71073$ $\AA)$ X-ray radiation or using a Copper monochromatized $(\lambda=1.54178 \AA)$ X-ray radiation. Continuous wave X-band ESR spectra were obtained with a Bruker ELEXSYS E500 spectrometer using a variable temperature Bruker liquid nitrogen cryostat.

\subsection{Synthetic procedures and characterization data}

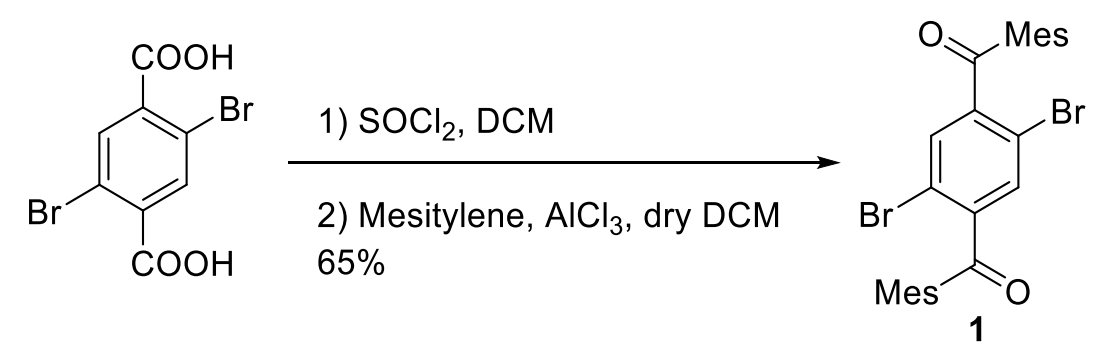

Synthesis of compound 1: 2,5-Dibromoterephthalic acid (3.23 g, $10.0 \mathrm{mmol})$ was suspended in dry DCM $(40 \mathrm{ml})$, and $\mathrm{SOCl}_{2}(2.25 \mathrm{ml}, 40.0 \mathrm{mmol})$ was added into the solution. The mixture was refluxed for $12 \mathrm{hrs}$ and then the solvent was removed under vacuum. The residue was dissolved in dry DCM (60 mL), and then mesitylene (7.2 g, $60.0 \mathrm{mmol})$ and $\mathrm{AlCl}_{3}(5.34 \mathrm{~g}, 40.0$ mmol) was added under ice bath. The mixture was gradually warmed to room temperature and then refluxed for overnight. The mixture was quenched by dilute hydrochloric acid (1M), and the organic layer was dried over anhydrous $\mathrm{Na}_{2} \mathrm{SO}_{4}$. The solvent was removed under vacuum and the residue was further washed with hexane to give crude product. The crude product was purified by column chromatography on silica gel with hexane/DCM $\left(2 / 1, \mathrm{v} / \mathrm{v}, R_{f}=0.3\right)$ as eluent 
to give pure product 1 as a white solid $\left(3.4 \mathrm{~g}, 65 \%\right.$ yield). ${ }^{1} \mathrm{H} \mathrm{NMR}\left(500 \mathrm{MHz}, \mathrm{CDCl}_{3}\right): \delta$ (ppm) $7.66(\mathrm{~s}, 2 \mathrm{H}), 6.89(\mathrm{~s}, 4 \mathrm{H}), 2.32(\mathrm{~s}, 6 \mathrm{H}), 2.14(\mathrm{~s}, 12 \mathrm{H}) ;{ }^{13} \mathrm{C} \mathrm{NMR}\left(125 \mathrm{MHz}, \mathrm{CDCl}_{3}\right): \delta(\mathrm{ppm})$ 197.44, 142.90, 140.52, 136.73, 135.77, 135.41, 129.25, 119.92, 21.22, 20.12; HRMS analysis (APCI): calcd for $\mathrm{C}_{26} \mathrm{H}_{25} \mathrm{Br}_{2} \mathrm{O}_{2}(\mathrm{M}+\mathrm{H})^{+}$: 527.0216; found: 527.0224 (error: +1.5 ppm).
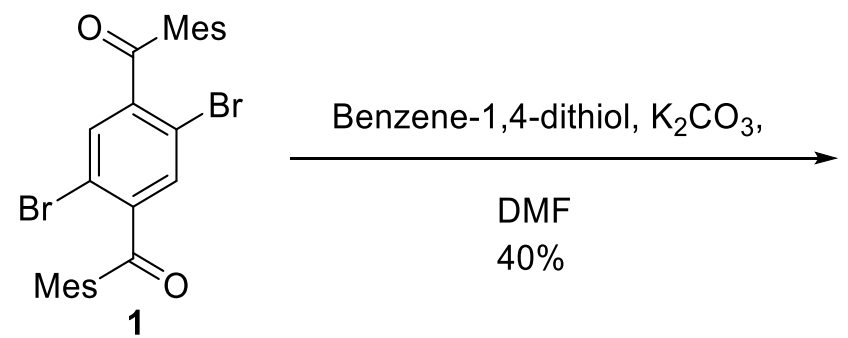<smiles>CC(=O)c1cc(Sc2ccc(Sc3cc(C(C)=O)c(Br)cc3C(C)=O)cc2)c(C(C)=O)cc1Br</smiles>

Synthesis of compound 2: Compound 1 (1.05 g, $2.0 \mathrm{mmol})$, benzene-1,4-dithiol (0.07 g, 0.5 $\mathrm{mmol})$ and $\mathrm{K}_{2} \mathrm{CO}_{3}(0.14 \mathrm{~g}, 1 \mathrm{mmol})$ were dissolved in dry DMF $(60 \mathrm{ml})$. The reaction mixture was stirred at $60{ }^{\circ} \mathrm{C}$ for overnight, and then cooled down to room temperature and quenched with $10 \%$ aqueous $\mathrm{HCl}$ solution, extracted by DCM. The organic layer was then collected, dried over $\mathrm{Na}_{2} \mathrm{SO}$. The solvent was removed and the crude product was purified by column chromatography on silica gel with hexane/DCM $\left(1 / 2, \mathrm{v} / \mathrm{v}, R_{f}=0.3\right)$ as eluent to give pure product 2 as a yellow solid $\left(0.21 \mathrm{~g}, 40 \%\right.$ yield). ${ }^{1} \mathrm{H} \mathrm{NMR}\left(500 \mathrm{MHz}, \mathrm{CDCl}_{3}\right): \delta(\mathrm{ppm}) 7.67(\mathrm{~s}$, 2H), $7.20(\mathrm{~s}, 4 \mathrm{H}), 6.96(\mathrm{~s}, 4 \mathrm{H}), 6.72(\mathrm{~s}, 4 \mathrm{H}), 6.62(\mathrm{~s}, 2 \mathrm{H}), 2.37(\mathrm{~s}, 6 \mathrm{H}), 2.35(\mathrm{~s}, 6 \mathrm{H}), 2.17(\mathrm{~s}$, $12 \mathrm{H}), 1.88(\mathrm{~s}, 12 \mathrm{H}) ;{ }^{13} \mathrm{C} \mathrm{NMR}\left(125 \mathrm{MHz}, \mathrm{CDCl}_{3}\right): \delta(\mathrm{ppm}) 199.33,198.40,142.57,141.18$, 139. $84,139.72,137.72,136.49,136.46,135.74,135.53,134.65,134.57,133.54,130.92$, 128.87, 128.58, 116.04, 21.33, 21.21, 19.53, 19.38; HRMS analysis (APCI): calcd for $\mathrm{C}_{58} \mathrm{H}_{53} \mathrm{Br}_{2} \mathrm{O}_{4} \mathrm{~S}_{2}(\mathrm{M}+\mathrm{H})^{+}$: 1035.1747; found: 1035.1725 (error: $-2.1 \mathrm{ppm}$ ).
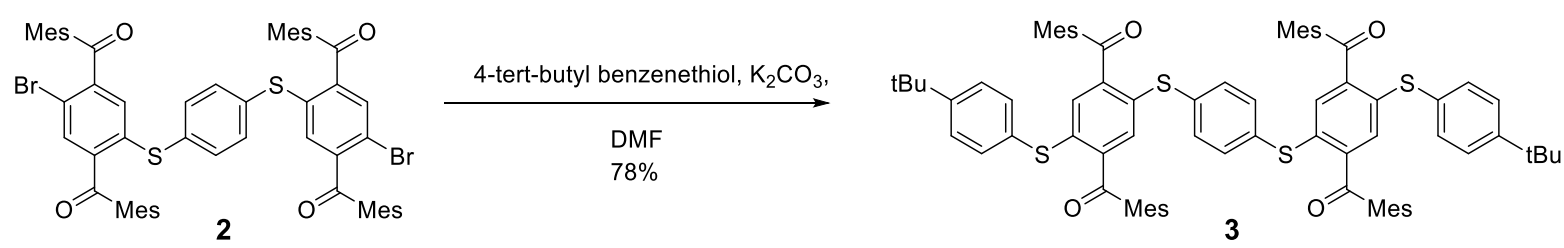

Synthesis of compound 3: 2 ( $0.10 \mathrm{~g}, 0.1 \mathrm{mmol})$, 4-tert-butyl benzenethiol ( $0.09 \mathrm{~g}, 0.5 \mathrm{mmol})$ and $\mathrm{K}_{2} \mathrm{CO}_{3}(0.14 \mathrm{~g}, 1 \mathrm{mmol})$ were dissolved in dry DMF $(40 \mathrm{ml})$. The reaction mixture was stirred at $60{ }^{\circ} \mathrm{C}$ for overnight, and then cooled down to room temperature and quenched with $10 \%$ aqueous $\mathrm{HCl}$ solution, extracted by DCM. The organic layer was then collected, dried over $\mathrm{Na}_{2} \mathrm{SO}_{4}$. The solvent was removed and the crude product was purified by column chromatography on silica gel with hexane/DCM $\left(1 / 2, \mathrm{v} / \mathrm{v}, R_{f}=0.3\right)$ as eluent to give pure product 3 as a yellow solid $\left(0.09 \mathrm{~g}, 78 \%\right.$ yield). ${ }^{1} \mathrm{H} \mathrm{NMR}\left(500 \mathrm{MHz}, \mathrm{CDCl}_{3}\right)$ : $\delta(\mathrm{ppm}) 7.28$ $7.25(\mathrm{~s}, 8 \mathrm{H}), 7.14(\mathrm{~s}, 4 \mathrm{H}), 7.07(\mathrm{~s}, 2 \mathrm{H}), 6.73(\mathrm{~s}, 4 \mathrm{H}), 6.70(\mathrm{~s}, 4 \mathrm{H}), 6.69(2 \mathrm{H}), 2.36(\mathrm{~s}, 6 \mathrm{H}), 2.30$ $(\mathrm{s}, 6 \mathrm{H}), 1.95(\mathrm{~s}, 12 \mathrm{H}), 1.88(\mathrm{~s}, 12 \mathrm{H}) 1.36(\mathrm{~s}, 18 \mathrm{H}) ;{ }^{13} \mathrm{C} \mathrm{NMR}\left(125 \mathrm{MHz}, \mathrm{CDCl}_{3}\right): \delta(\mathrm{ppm})$ 200.73, 200.31, 152.51, 139.81, 139.11, 138.67, 137.73, 136.72, 136.29, 136.19, 136.10, 135.94, 134.20, 133.87, 131.74, 131.30, 128.37, 128.26, 126.55, 34.69, 31.26, 21.38, 21.25, 19.19, 19.10; HRMS analysis (APCI): calcd for $\mathrm{C}_{78} \mathrm{H}_{79} \mathrm{O}_{4} \mathrm{~S}_{4}(\mathrm{M}+\mathrm{H})^{+}$: 1207.4856; found: 1207.4853 (error: $-0.2 \mathrm{ppm}$ ). 


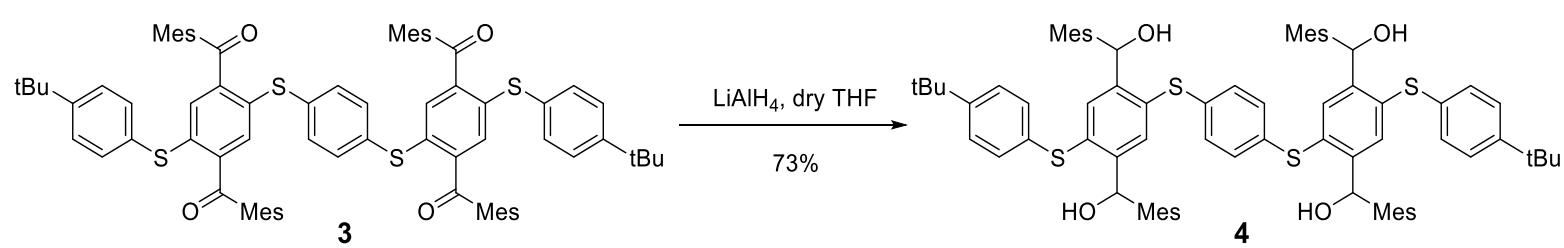

Synthesis of compound 4: Lithium aluminum hydride (95 mg, $2.5 \mathrm{mmol}$ ) was dissolved in 20 $\mathrm{mL}$ dry THF, a solution of $\mathbf{3}$ (121 mg, $0.1 \mathrm{mmol})$ in $10 \mathrm{~mL}$ dry THF was injected into the system dropwisely at room temperature. The reaction mixture was stirred for $12 \mathrm{hrs}$ and quenched with $\mathrm{Na}_{2} \mathrm{SO}_{4} \cdot 10 \mathrm{H}_{2} \mathrm{O}$. The organic layer was extracted with diethyl ether, washed three times with brine, and then dried with anhydrous $\mathrm{Na}_{2} \mathrm{SO}_{4}$. After evaporating the solvent, a light yellow solid 4 (89 mg, 73\% yield) was obtained by simply washing with hexane as crude product. No clean NMR was obtained as the isomers cannot be seperated by column chromatography. The crude product 4 was used directly for the next step. HR MS analysis (APCI): calcd for $\mathrm{C}_{78} \mathrm{H}_{86} \mathrm{O}_{4} \mathrm{~S}_{4}$ $(\mathrm{M})^{+}$: 1214.5403; found: 1214.5405 (error: $+0.2 \mathrm{ppm}$ ).

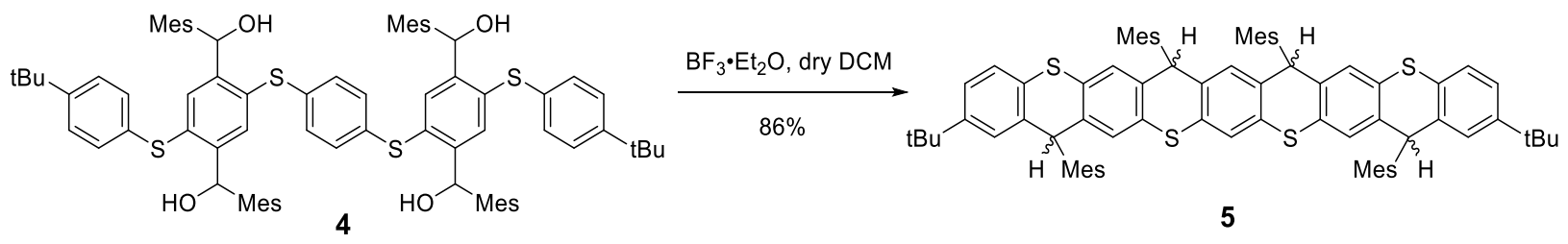

Synthesis of compound 5: Compound 4 (90 $\mathrm{mg}, 0.074 \mathrm{mmol})$ was dissolved in dry DCM (30 $\mathrm{ml})$ in ice bath, boron trifluoride diethyl etherate $(0.10 \mathrm{ml}, 0.8 \mathrm{mmol})$ was added dropwisely into the solution. The reaction mixture was stirred for $2 \mathrm{hrs}$, then quenched with water. The organic layer was washed with brine and dried with anhydrous $\mathrm{Na}_{2} \mathrm{SO}_{4}$. After removal of the solvent under vacuum, the crude product was purified by a flash column chromatography on silica gel with hexane/DCM $\left(4 / 1, \mathrm{v} / \mathrm{v}, R_{f}=0.8\right)$ as eluent to give the product $5(72 \mathrm{mg}, 86 \%$ yield) as a pale purple flaky solid. The purple colour was ascribed to the trace partially oxidation of compound 5 during elution, which losing two hydrogen. As such, no clean ${ }^{1} \mathrm{H}$ and ${ }^{13} \mathrm{C}$ NMR spectra were obtainable. HR MS (APCI): calcd for $\mathrm{C}_{78} \mathrm{H}_{79} \mathrm{~S}_{4}(\mathrm{M}+\mathrm{H})^{+}, 1143.5059$; found, 1143.5042 (error: $-1.5 \mathrm{ppm}$ ).

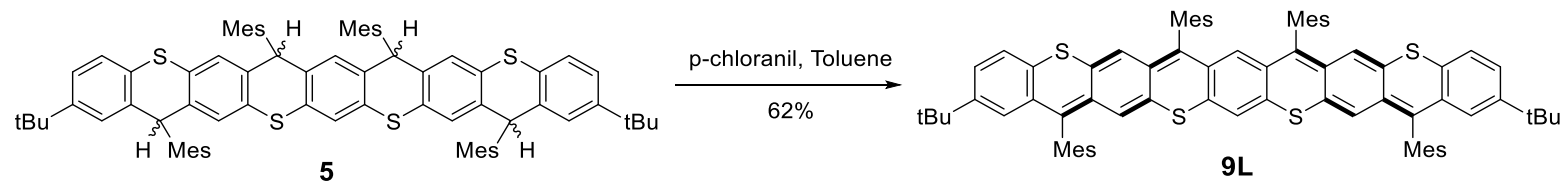

Synthesis of compound 9L: Compound 5 (72 $\mathrm{mg}, 0.064 \mathrm{mmol})$ was dissolved in dry toluene $(30 \mathrm{ml})$, then $p$-chloranil $(78 \mathrm{mg}, 0.32 \mathrm{mmol})$ in toluene was added dropwisely into the solution, and the reaction was stirred at $90{ }^{\circ} \mathrm{C}$ for 3 days. The solution was extracted with dichloromethane and the organic layer was washed with brine and dried over anhydrous sodium sulfate. The solvent was removed and the residue was purified by column chromatography on silica gel with hexane/DCM (3/1, v/v) as eluent to give compound 9L as a dark blue solid (45 mg, 62\% yield). ${ }^{1} \mathrm{H}$ NMR (500 MHz, $\left.\mathrm{C}_{6} \mathrm{D}_{6}\right): \delta(\mathrm{ppm}) 6.83(\mathrm{~s}, 4 \mathrm{H}), 6.78-6.58(\mathrm{~m}, 6 \mathrm{H}), 6.52$ (s, 
4H), 6.30-6.10 (m, 5H), $5.52(\mathrm{~s}, 1 \mathrm{H}), 2.14(\mathrm{~s}, 12 \mathrm{H}), 2.04(\mathrm{~s}, 12 \mathrm{H}), 1.83(\mathrm{~s}, 12 \mathrm{H}), 0.93(\mathrm{~s}, 18 \mathrm{H})$;

${ }^{13} \mathrm{C}$ NMR spectrum is not available even the scan is more than 24 hours, only partial signals appear. HRMS analysis (APCI): calcd for $\mathrm{C}_{78} \mathrm{H}_{75} \mathrm{~S}_{4}(\mathrm{M}+\mathrm{H})^{+}$: 1139.4746; found: 1139.4752 (error: $+0.5 \mathrm{ppm}$ ).

\subsection{Chemical Oxidation of 9L}

Typical oxidation procedure to radical cation, dication, radical trication and tetracation of 9L:

$\mathrm{NO} \cdot \mathrm{SbF}_{6}(0.01 \mathrm{mmol}$ for radical cation, $0.02 \mathrm{mmol}$ for dication, $0.03 \mathrm{mmol}$ for radical trication and $0.04 \mathrm{mmol}$ for tetracation) dissolved in acetonitrile $(50 \mu \mathrm{l})$ was added into the dry DCM solution of 9L ( $0.01 \mathrm{mmol})$, respectively. The oxidized compounds were formed in $2 \mathrm{mins}$, and the solvent was removed under vacuum to give the radical cation, dication, radical trication and tetracation without further purification.

9L ${ }^{4+}:{ }^{1} \mathrm{H}$ NMR (500 MHz, $\left.\mathrm{CD}_{2} \mathrm{Cl}_{2}\right): \delta(\mathrm{ppm}) 8.68(\mathrm{~s}, 2 \mathrm{H}), 8.66(\mathrm{~d}, J=8.9 \mathrm{~Hz}, 2 \mathrm{H}), 8.55(\mathrm{~s}, 2 \mathrm{H})$, $8.52\left(\mathrm{dd},{ }^{3} \mathrm{~J}=9.0 \mathrm{~Hz},{ }^{4} \mathrm{~J}=2.0 \mathrm{~Hz}, 2 \mathrm{H}\right), 8.04$ (two singlets, $\left.3 \mathrm{H}\right), 7.76(\mathrm{~s}, 1 \mathrm{H}), 7.28(\mathrm{~s}, 4 \mathrm{H}), 7.08$ $(\mathrm{s}, 2 \mathrm{H}), 7.00(\mathrm{~s}, 2 \mathrm{H}), 2.55(\mathrm{~s}, 6 \mathrm{H}), 2.47(\mathrm{~s}, 6 \mathrm{H}), 1.96(\mathrm{~s}, 12 \mathrm{H}), 1.76-1.75$ (two singlets, 12H), $1.30(\mathrm{~s}, 18 \mathrm{H})$.

\section{Additional spectra}

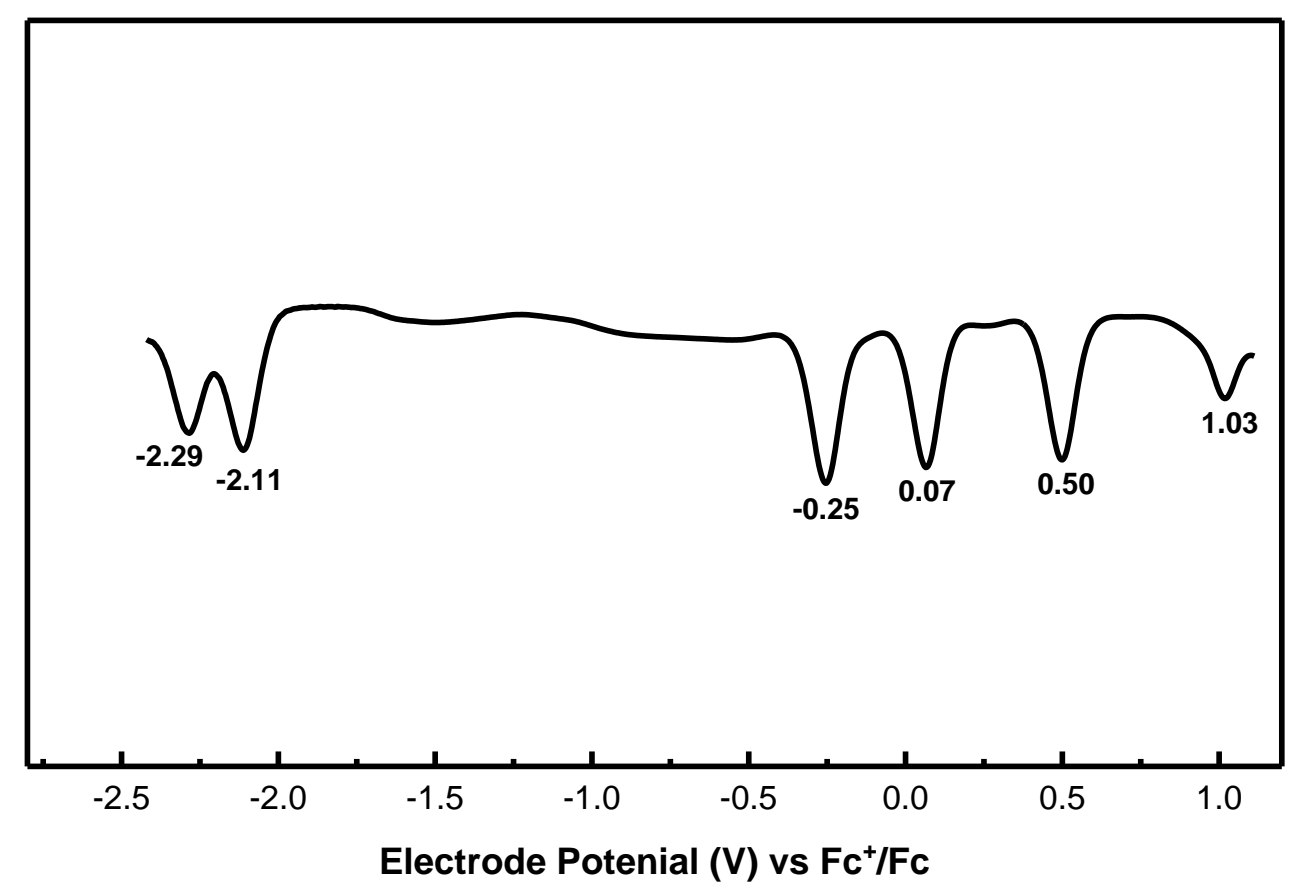

Figure S1. Differential pulse voltammogram $\left(1 \times 10^{-3} \mathrm{M}\right)$ of $\mathbf{9 L}$ in dry dichloromethane (DCM) with $0.1 \mathrm{M} \mathrm{Bu}_{4} \mathrm{NPF}_{6}$ as the supporting electrolyte, $\mathrm{AgCl} / \mathrm{Ag}$ as reference electrode, $\mathrm{Au}$ as working electrode (surface area $=12.6 \mathrm{~mm}^{2}$ ), Pt wire as counter electrode (potential step = $0.004 \mathrm{~V}$, pulse amplitude $=0.05 \mathrm{~V}$, pulse width $=0.05 \mathrm{~s}$ in the oxidation mode). The electrode potential was externally calibrated by $\mathrm{Fc}^{+} / \mathrm{Fc}$ couple. 


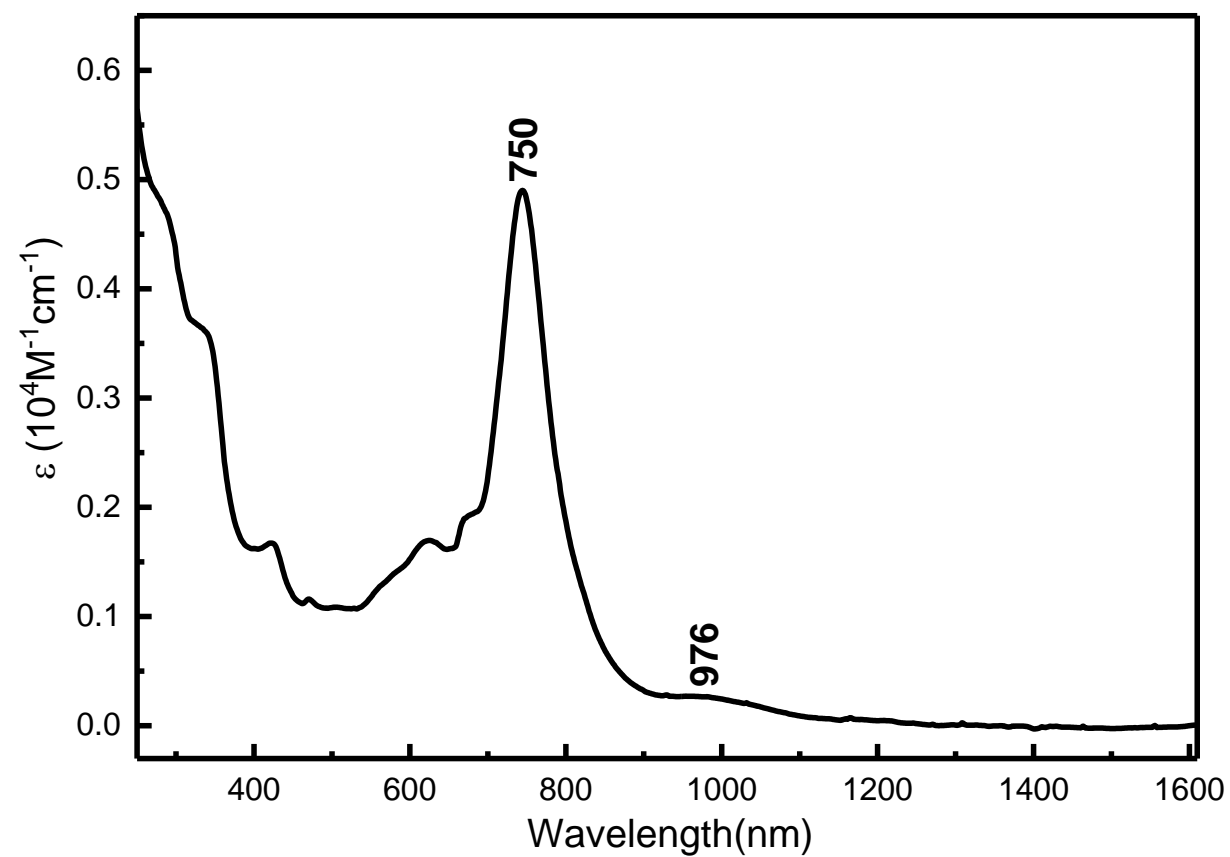

Figure S2. UV-Vis-NIR absorption spectra of pure $\mathbf{9 L}^{4+}$ in dry DCM.
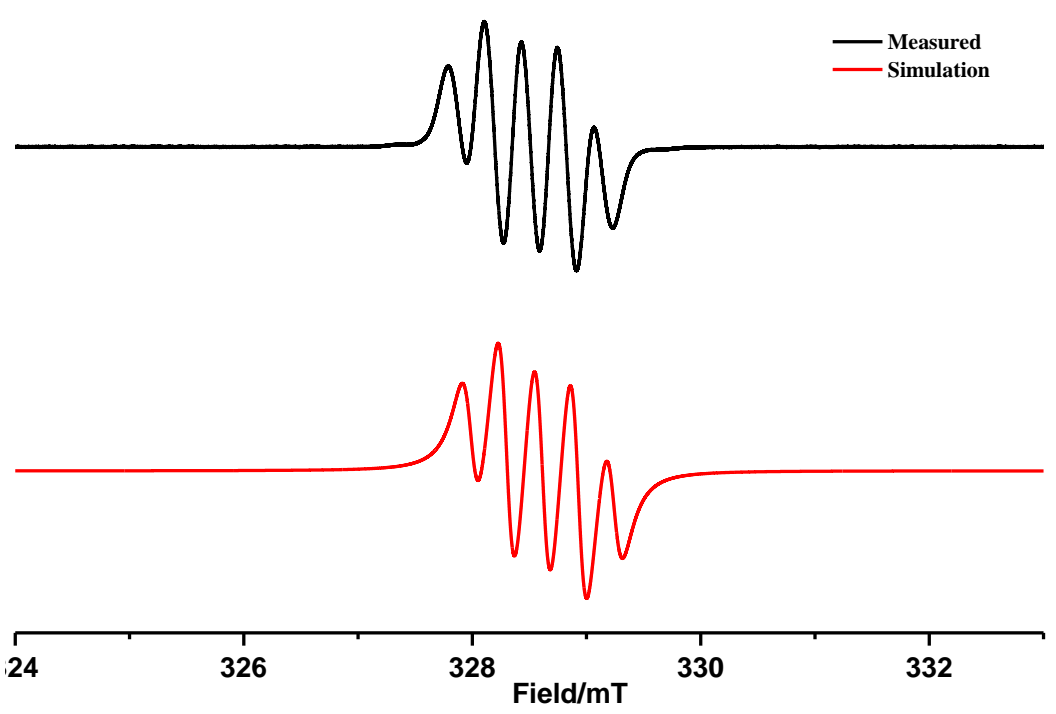

Figure S3. Measured and simulated ESR spectrum of $9 \mathrm{~L}^{\boldsymbol{\bullet}^{2+}}, \mathrm{g}=2.00357, \mathrm{Hfcc}_{1}=0.628 \mathrm{mT}$, $\mathrm{Hfcc}_{2}=0.311 \mathrm{mT}$, line-width $=0.167 \mathrm{mT}$. 


\section{DFT calculations}

Density functional theory calculations were employed with Gaussian 09 package, ${ }^{1}$ utilizing the UCAM-B3LYP ${ }^{2-4}$ level of theory with Pople basis set $6-31 \mathrm{G}^{* 5-7}$ for all molecules in the gas phase. NICS values were calculated using the standard GIAO procedure (NMR pop $=$ NCSall). ${ }^{8}$ ACID plots was calculated by using the method developed by Herges. ${ }^{9}$

Table S1. Selected TD-DFT (B3LYP/6-31G*) calculated energies, oscillator strength and compositions of major electronic transitions of $\mathbf{9 L}$.

\begin{tabular}{ccc}
\hline $\begin{array}{c}\text { Wavelength } \\
(\mathbf{n m})\end{array}$ & Osc. Strength $(\boldsymbol{f})$ & Major contributions \\
\hline 310.0 & 0.1086 & $\mathrm{HOMO} \rightarrow \mathrm{L}+15(82 \%)$ \\
328.1 & 0.2878 & $\mathrm{H}-6 \rightarrow \mathrm{L}+1(44 \%), \mathrm{HOMO} \rightarrow \mathrm{L}+9(25 \%)$ \\
& & $\mathrm{H}-3 \rightarrow \mathrm{LUMO}(17 \%), \mathrm{H}-2 \rightarrow \mathrm{L}+1(33 \%)$, \\
384.9 & 0.3039 & $\mathrm{H}-1 \rightarrow \mathrm{L}+2(15 \%)$ \\
546.9 & 1.2599 & $\mathrm{H}-1 \rightarrow \mathrm{L}+1(93 \%)$ \\
689.2 & 1.261 & $\mathrm{HOMO} \rightarrow \mathrm{LUMO}(94 \%)$ \\
\hline
\end{tabular}

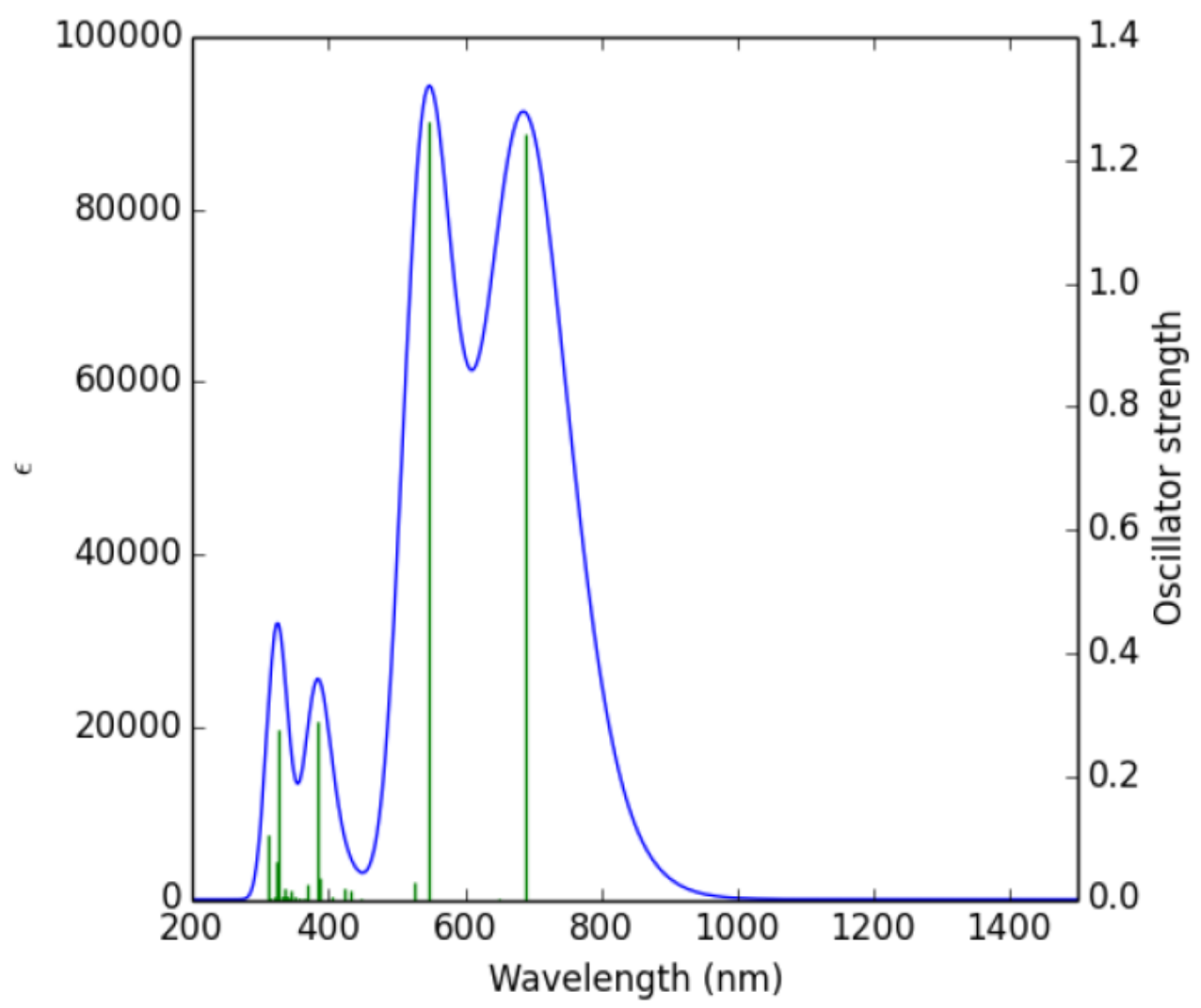

Figure S4. Calculated (B3LYP/6-31G*) absorption spectrum of 9L. 
Table S2. Selected TD-DFT (B3LYP/6-31G*) calculated energies, oscillator strength and compositions of major electronic transitions of $9 \mathbf{L}^{\bullet+}$.

\begin{tabular}{ccc}
\hline $\begin{array}{c}\text { Wavelength } \\
(\mathbf{n m})\end{array}$ & Osc. Strength & Major contributions \\
\hline 573.3 & 0.1529 & $\mathrm{H}-11(\mathrm{~B}) \rightarrow \mathrm{LUMO}(\mathrm{B})\left(\begin{array}{c}(47 \%), \mathrm{H}-9(\mathrm{~B}) \rightarrow \mathrm{LUMO}(\mathrm{B}) \\
(12 \%)\end{array}\right.$ \\
609.2 & 0.9745 & $\mathrm{H}-1(\mathrm{~A}) \rightarrow \mathrm{L}+1(\mathrm{~A})\left(\begin{array}{c}(28 \%), \mathrm{HOMO}(\mathrm{B}) \rightarrow \mathrm{L}+2(\mathrm{~B}) \\
(54 \%)\end{array}\right.$ \\
712 & 0.1915 & $\mathrm{H}-1(\mathrm{~A}) \rightarrow \mathrm{L}+1(\mathrm{~A})\left(\begin{array}{c}(45 \%), \mathrm{HOMO}(\mathrm{A}) \rightarrow \mathrm{LUMO}(\mathrm{A}) \\
(33 \%)\end{array}\right.$ \\
798.5 & 0.0523 & $\mathrm{H}-1(\mathrm{~B}) \rightarrow \mathrm{LUMO}(\mathrm{B})(86 \%)$ \\
1066.9 & 0.4836 & $\mathrm{H}-1(\mathrm{~A}) \rightarrow \mathrm{L}+1(\mathrm{~A})(14 \%), \mathrm{HOMO}(\mathrm{A}) \rightarrow \mathrm{LUMO}(\mathrm{A})$ \\
1830.2 & 0.2597 & $\begin{array}{c}49 \%), \mathrm{HOMO}(\mathrm{B}) \rightarrow \mathrm{L}+2(\mathrm{~B})(27 \%) \\
\mathrm{HOMO}(\mathrm{B}) \rightarrow \mathrm{LUMO}(\mathrm{B})(93 \%)\end{array}$ \\
\hline
\end{tabular}

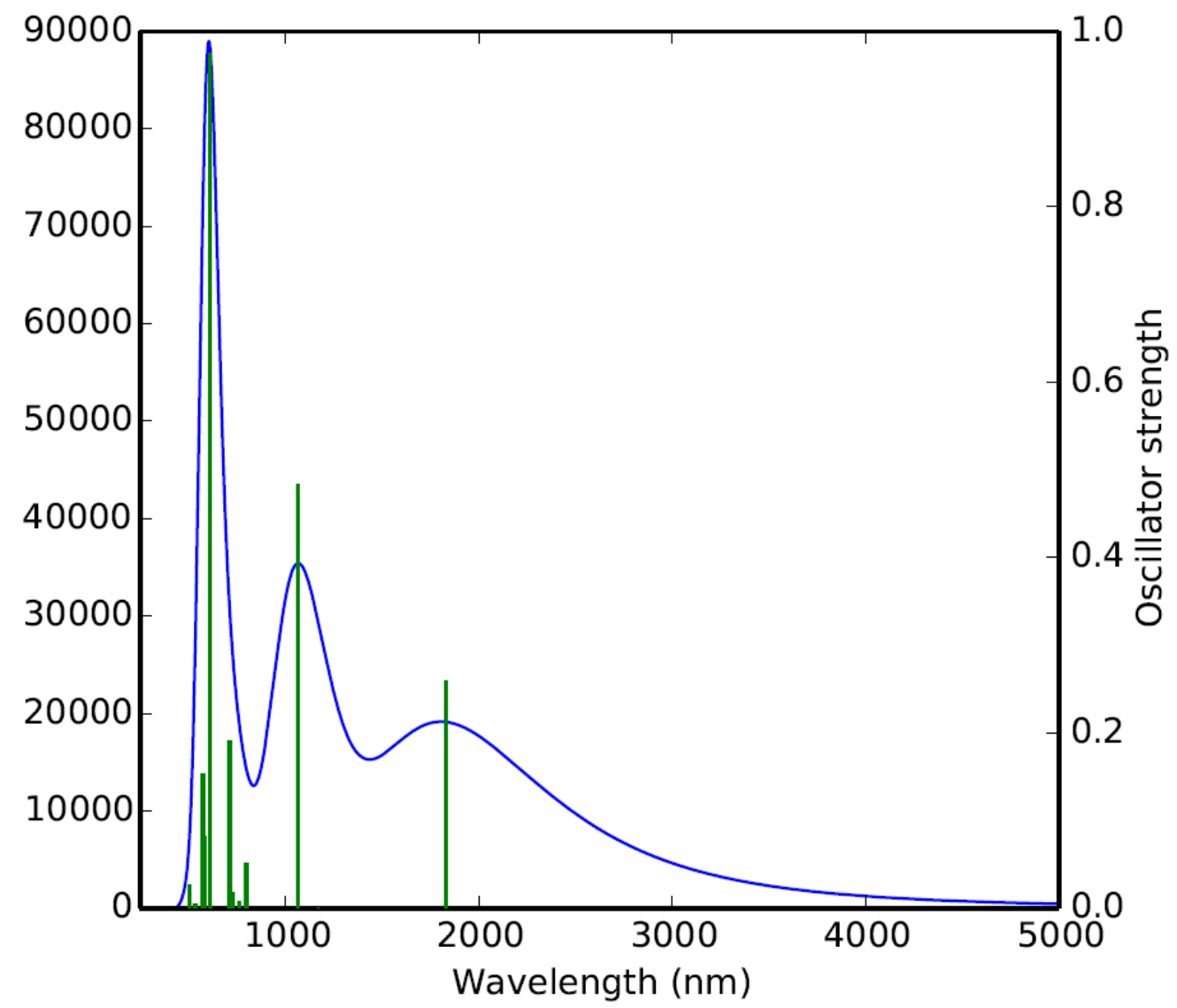

Figure S5. Calculated (UB3LYP/6-31G*) absorption spectrum of $\mathbf{9 L}^{\mathbf{*}}$. 
Table S3. Selected TD-DFT (B3LYP/6-31G*) calculated energies, oscillator strength and compositions of major electronic transitions of $\mathbf{9} \mathbf{L}^{2+}$.

\begin{tabular}{ccc}
\hline $\begin{array}{c}\text { Wavelength } \\
(\mathbf{n m})\end{array}$ & Osc. Strength $(f)$ & Major contributions \\
\hline 404.4 & 0.1355 & $\mathrm{H}-15 \rightarrow \mathrm{LUMO}(14 \%), \mathrm{H}-9 \rightarrow \mathrm{L}+1(28 \%)$, \\
& & $\mathrm{H}-6 \rightarrow \mathrm{L}+2(32 \%)$ \\
412.5 & 0.6667 & $\mathrm{H}-14 \rightarrow \mathrm{LUMO}(70 \%), \mathrm{H}-6 \rightarrow \mathrm{L}+2(14 \%)$ \\
& & $\mathrm{H}-9 \rightarrow \mathrm{L}+1(21 \%), \mathrm{H}-6 \rightarrow \mathrm{L}+2(34 \%)$, \\
445.7 & 0.1309 & $\mathrm{HOMO} \rightarrow \mathrm{L}+3(39 \%)$ \\
546.3 & 0.4793 & $\mathrm{H}-11 \rightarrow \mathrm{LUMO}(95 \%)$ \\
674.3 & 1.0179 & $\mathrm{H}-6 \rightarrow \mathrm{LUMO}(10 \%), \mathrm{HOMO} \rightarrow \mathrm{L}+2(82 \%)$ \\
1412.5 & 0.6278 & $\mathrm{HOMO} \rightarrow \mathrm{LUMO}(107 \%)$ \\
\hline
\end{tabular}

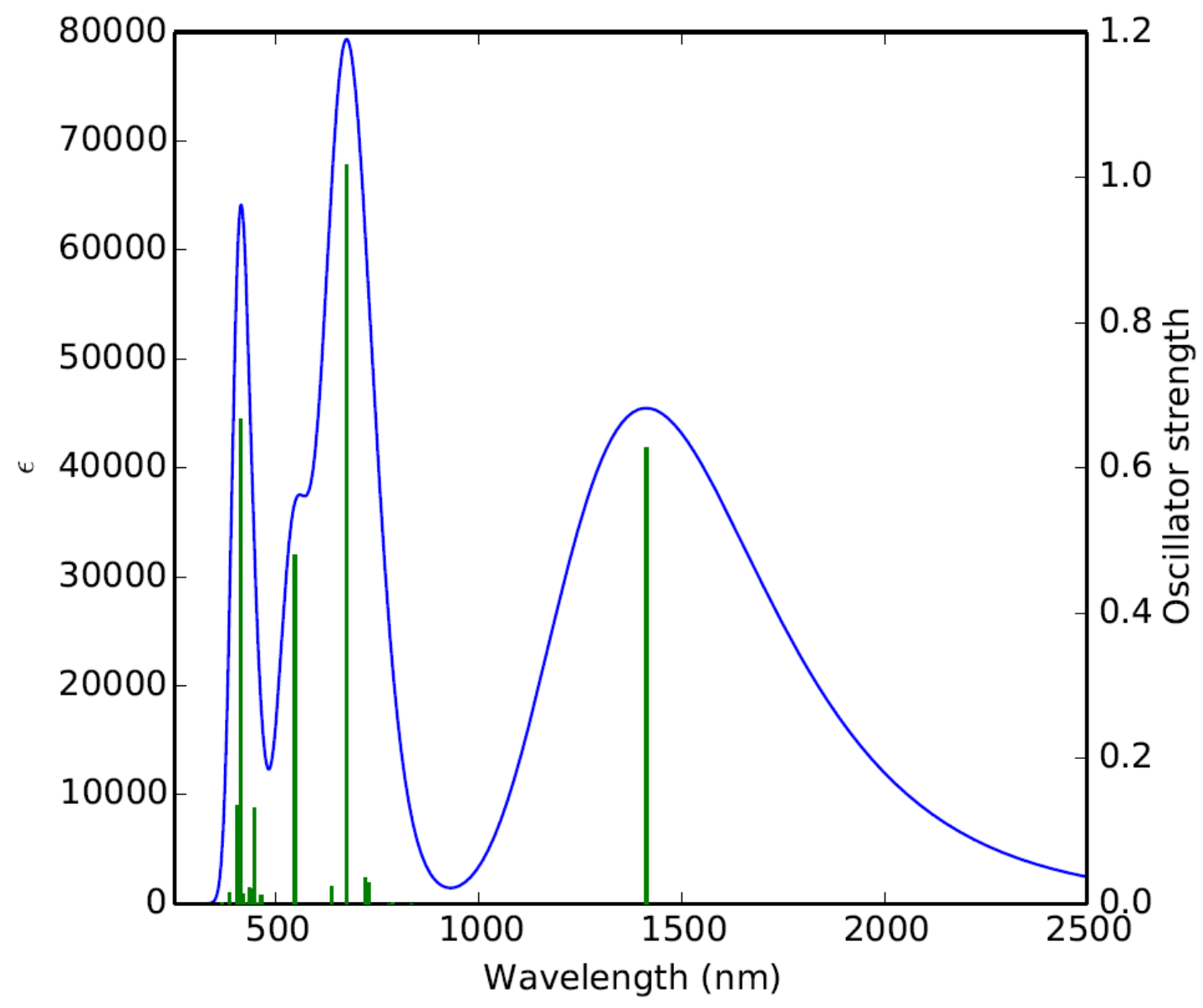

Figure S6. Calculated (B3LYP/6-31G*) absorption spectrum of $9 \mathbf{L}^{\mathbf{2 +}}$. 
Table S4. Selected TD-DFT (B3LYP/6-31G*) calculated energies, oscillator strength and compositions of major electronic transitions of $9 \mathbf{L}^{\cdot 3+}$.

\begin{tabular}{ccc}
\hline $\begin{array}{c}\text { Wavelength } \\
(\mathbf{n m})\end{array}$ & Osc. Strength & Major contributions \\
\hline 1021.6 & 0.0018 & $\mathrm{H}-4(\mathrm{~B}) \rightarrow \mathrm{LUMO}(\mathrm{B})(92 \%)$ \\
1041.8 & 0.0236 & $\begin{array}{c}\mathrm{H}-10(\mathrm{~A}) \rightarrow \mathrm{LUMO}(\mathrm{A})(27 \%), \mathrm{H}-9(\mathrm{~B}) \rightarrow \mathrm{LUMO}(\mathrm{B}) \\
(21 \%), \mathrm{H}-9(\mathrm{~B}) \rightarrow \mathrm{L}+1(\mathrm{~B})(21 \%), \mathrm{H}-\end{array}$ \\
& & $8(\mathrm{~B}) \rightarrow \mathrm{LUMO}(\mathrm{B})(14 \%)$ \\
1099.3 & 0.082 & $\mathrm{H}-9(\mathrm{~A}) \rightarrow \mathrm{LUMO}(\mathrm{A})(34 \%), \mathrm{H}-8(\mathrm{~B}) \rightarrow \mathrm{LUMO}(\mathrm{B})$ \\
1545.5 & 0.2764 & $(23 \%), \mathrm{H}-8(\mathrm{~B}) \rightarrow \mathrm{L}+1(\mathrm{~B})(24 \%)$ \\
& & $\mathrm{HOMO}(\mathrm{A}) \rightarrow \mathrm{LUMO}(\mathrm{A})(91 \%)$
\end{tabular}

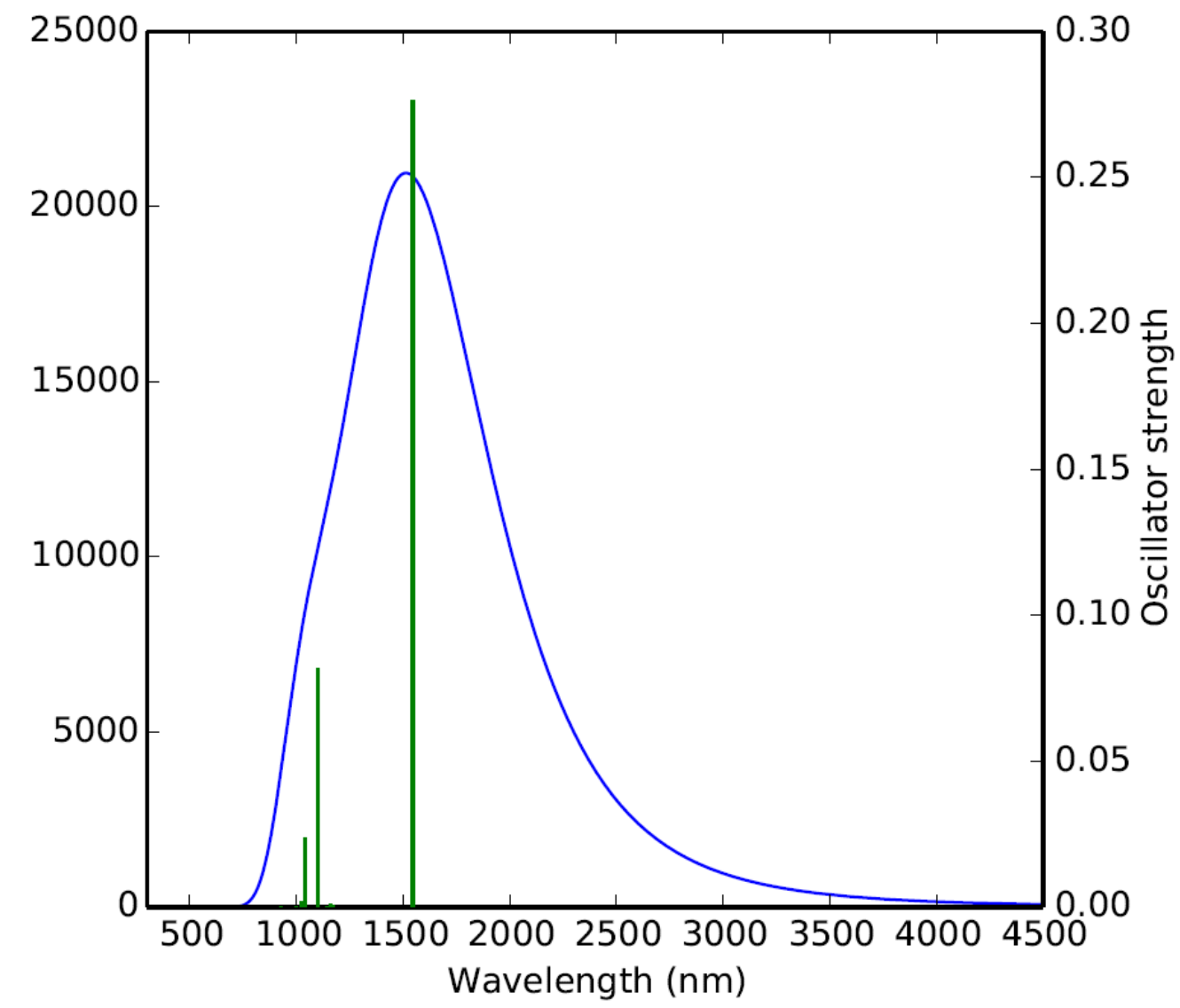

Figure S7. Calculated (UB3LYP/6-31G*) absorption spectrum of $\mathbf{9 \mathbf { L } ^ { \bullet 3 + }}$. 
Table S5. Selected TD-DFT (B3LYP/6-31G*) calculated energies, oscillator strength and compositions of major electronic transitions of $9 \mathbf{L}^{\mathbf{4 +}}$.

\begin{tabular}{ccc}
\hline $\begin{array}{c}\text { Wavelength } \\
(\mathbf{n m})\end{array}$ & Osc. Strength $(\boldsymbol{f})$ & Major contributions \\
\hline 533.1 & 0.7549 & $\mathrm{H}-15 \rightarrow$ LUMO $(15 \%), \mathrm{H}-10 \rightarrow \mathrm{L}+1(79 \%)$ \\
& & $\mathrm{H}-15 \rightarrow$ LUMO $(48 \%), \mathrm{H}-10 \rightarrow \mathrm{L}+1(11 \%)$, \\
567.2 & 0.1836 & HOMO $\rightarrow \mathrm{L}+2(27 \%)$ \\
623.1 & 0.2237 & $\mathrm{H}-11 \rightarrow$ LUMO $(93 \%), \mathrm{H}-12 \rightarrow$ LUMO $(3 \%)$ \\
1196.1 & 0.1616 & $\mathrm{H}-6 \rightarrow$ LUMO $(10 \%), \mathrm{H}-5 \rightarrow$ LUMO $(66 \%)$ \\
\hline
\end{tabular}

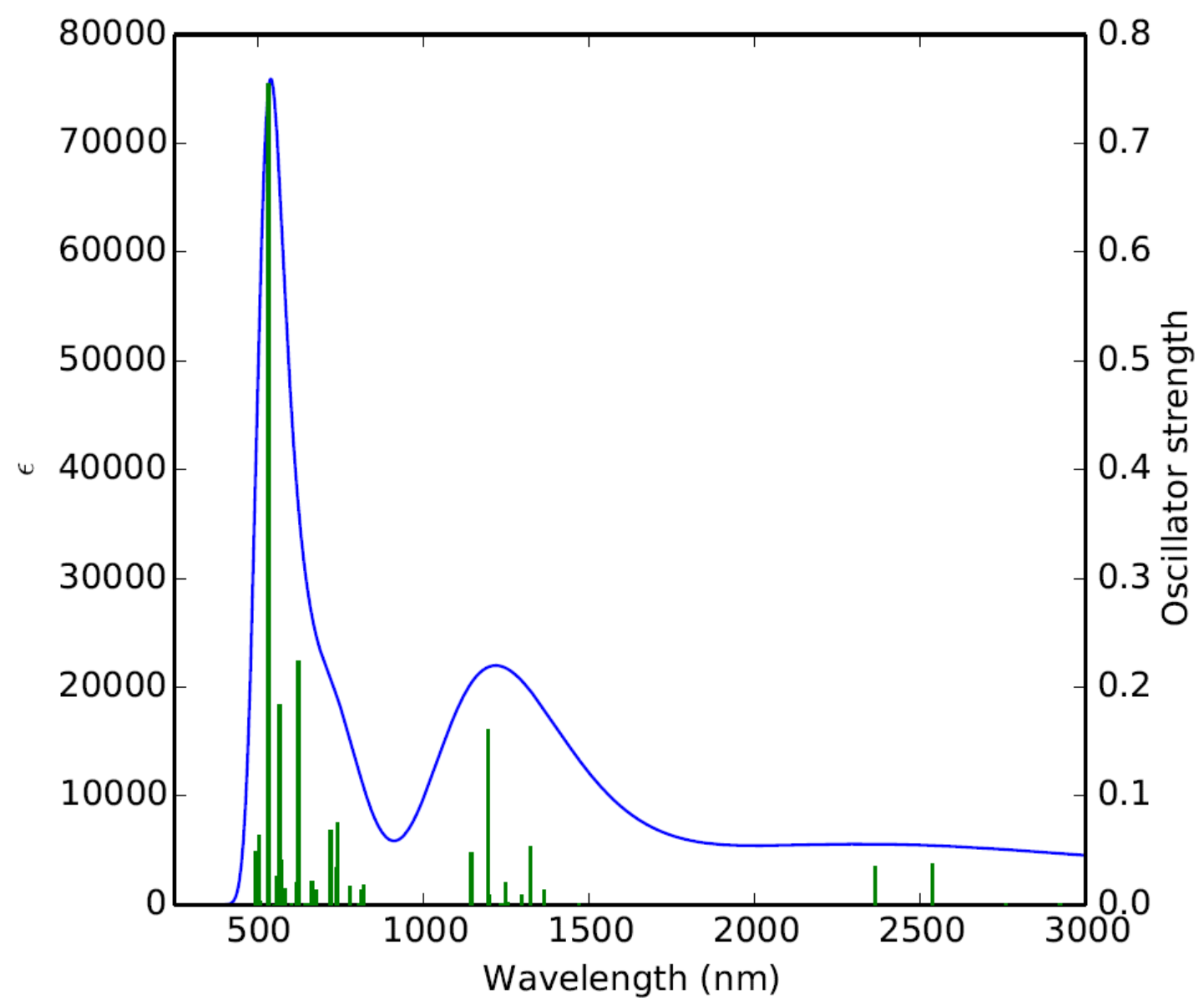

Figure S8. Calculated (B3LYP/6-31G*) absorption spectrum of $9 \mathbf{L}^{\mathbf{4 +}}$. 


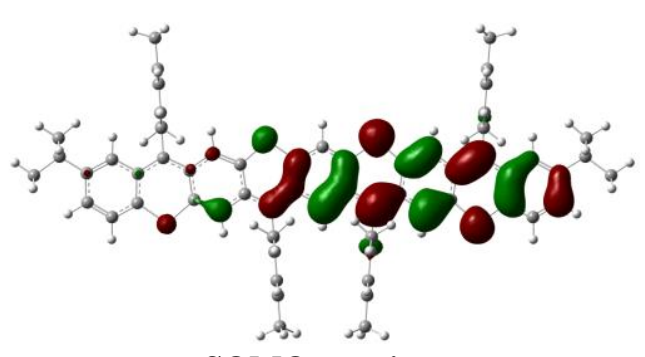

SOMO $\alpha$-spin

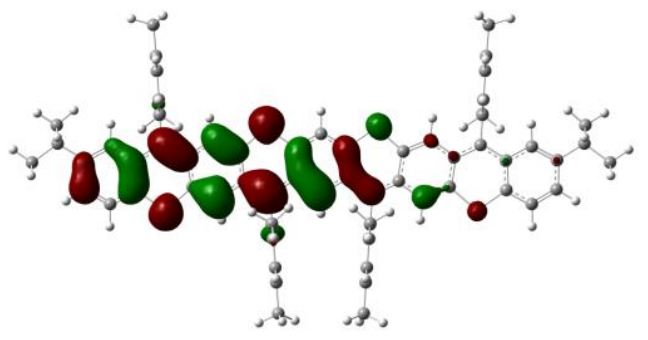

SOMO $\beta$-spin

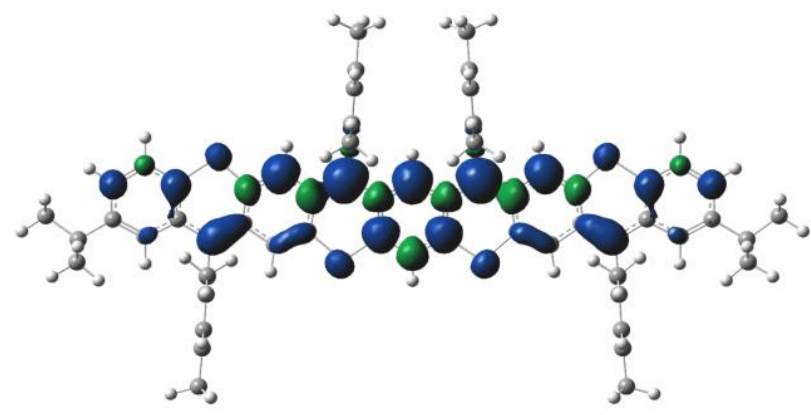

Spin density distribution

Figure S9. Calculated (UCAM-B3LYP/6-31G (d, p)) SOMOs for the $\alpha$ and $\beta$ electrons and spin density distribution of $\mathbf{9 L} \mathbf{L}^{\cdot 2+}$.

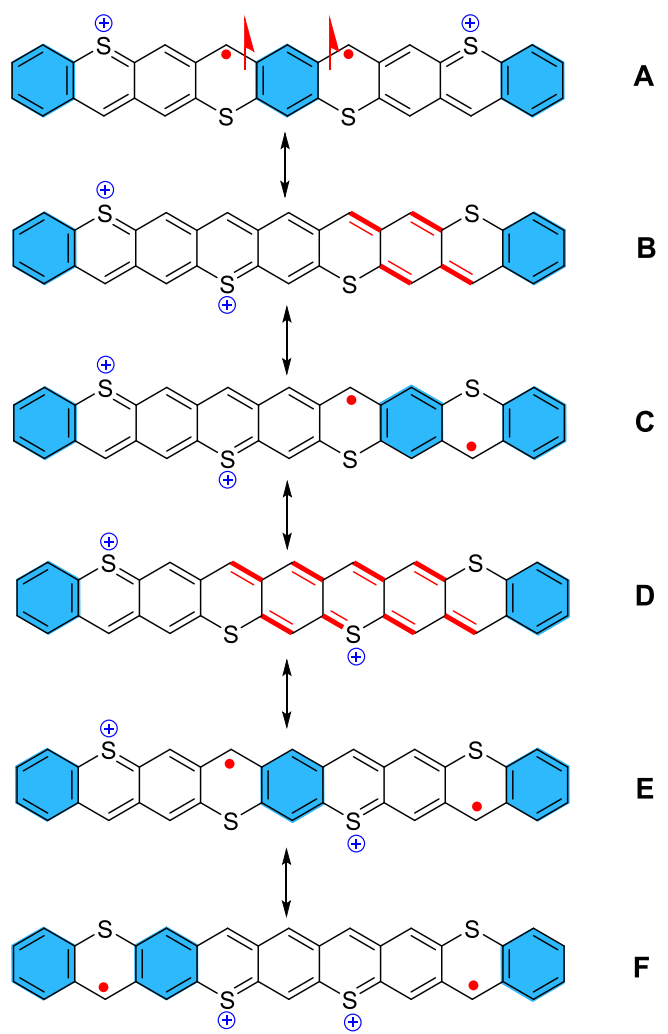

Figure S10. The resonance structures of $\mathbf{9 L} \cdot \boldsymbol{\bullet ⿻}^{+2+}$ (Note: ESR intensity increased with decreasing temperature for $\mathbf{9} \mathbf{L}^{\boldsymbol{\bullet}^{2+}}$ (see Figure 4 and Figure S3), but it did not show NMR signal from room temperature to $-80^{\circ} \mathrm{C}$. Combination of these experimental observations and spin density distribution map (Figure 5) reveals that the triplet diradical resonance form A mainly contributes the dicationic state of $\mathbf{9 L}$. 

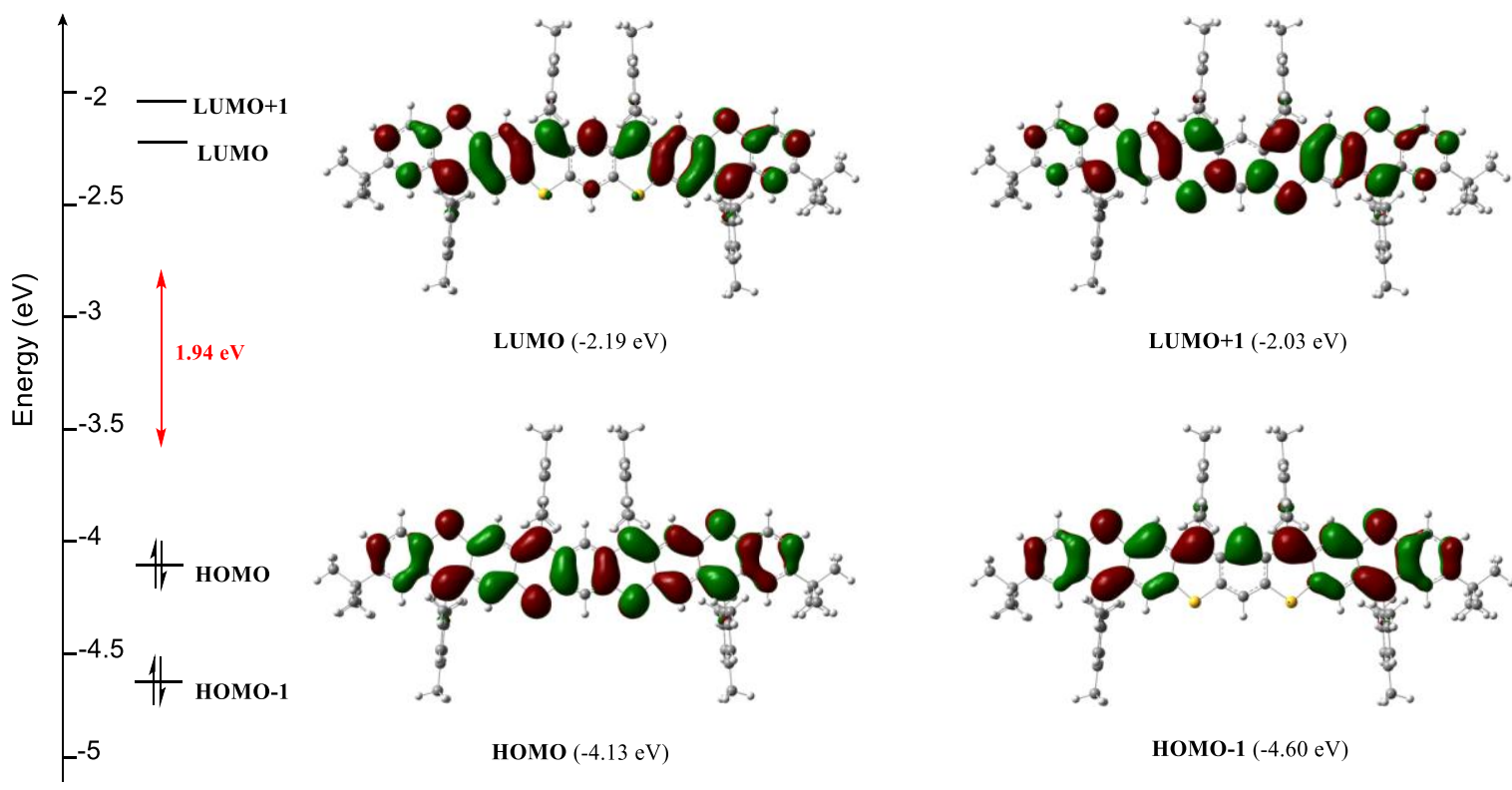

Figure S11. Selected calculated (B3LYP/6-31G*) frontier molecular orbital profiles of 9L.
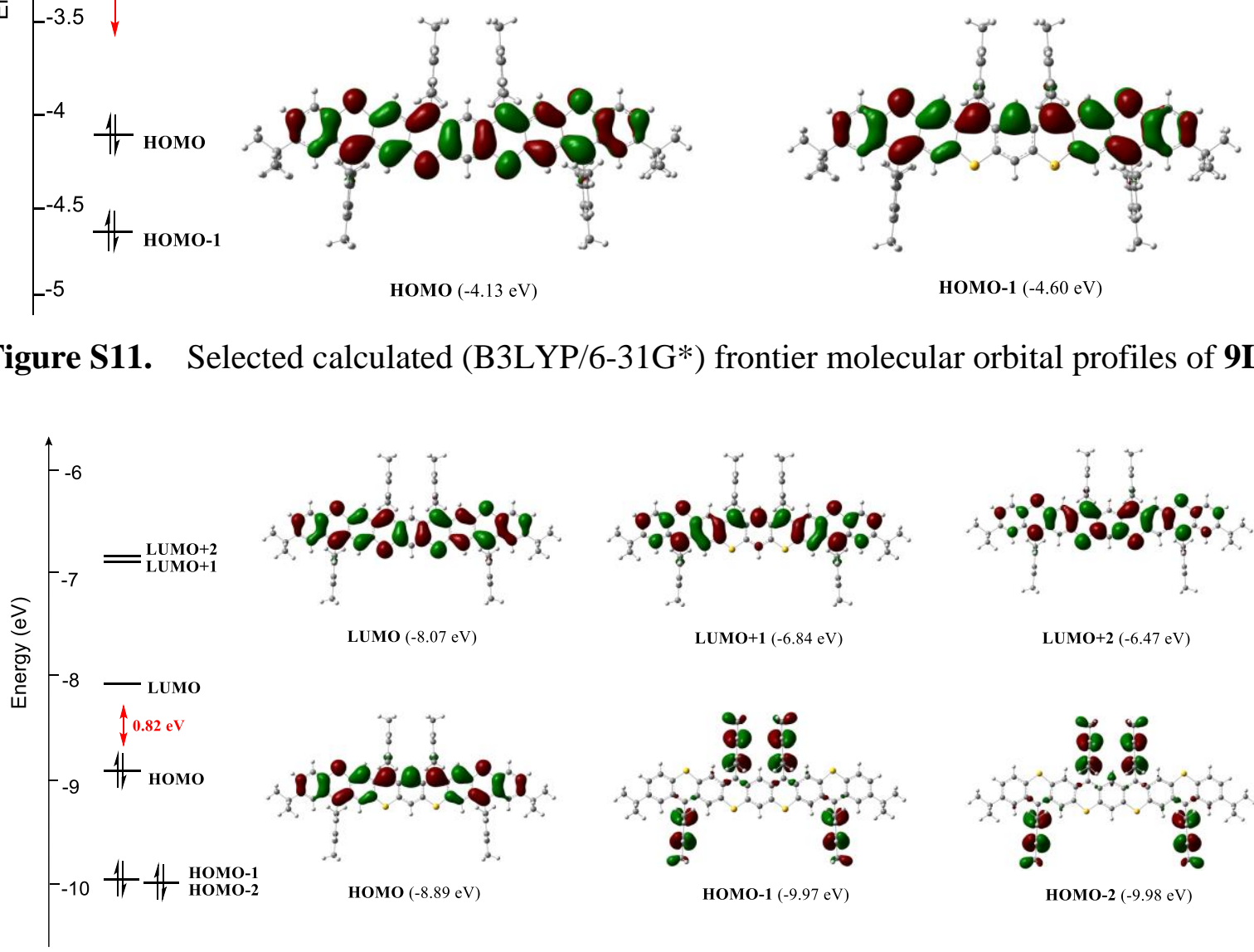

Figure S12. Selected calculated (B3LYP/6-31G*) frontier molecular orbital profiles of $9 \mathbf{L}^{\boldsymbol{\bullet \cdot 2 +}}$.
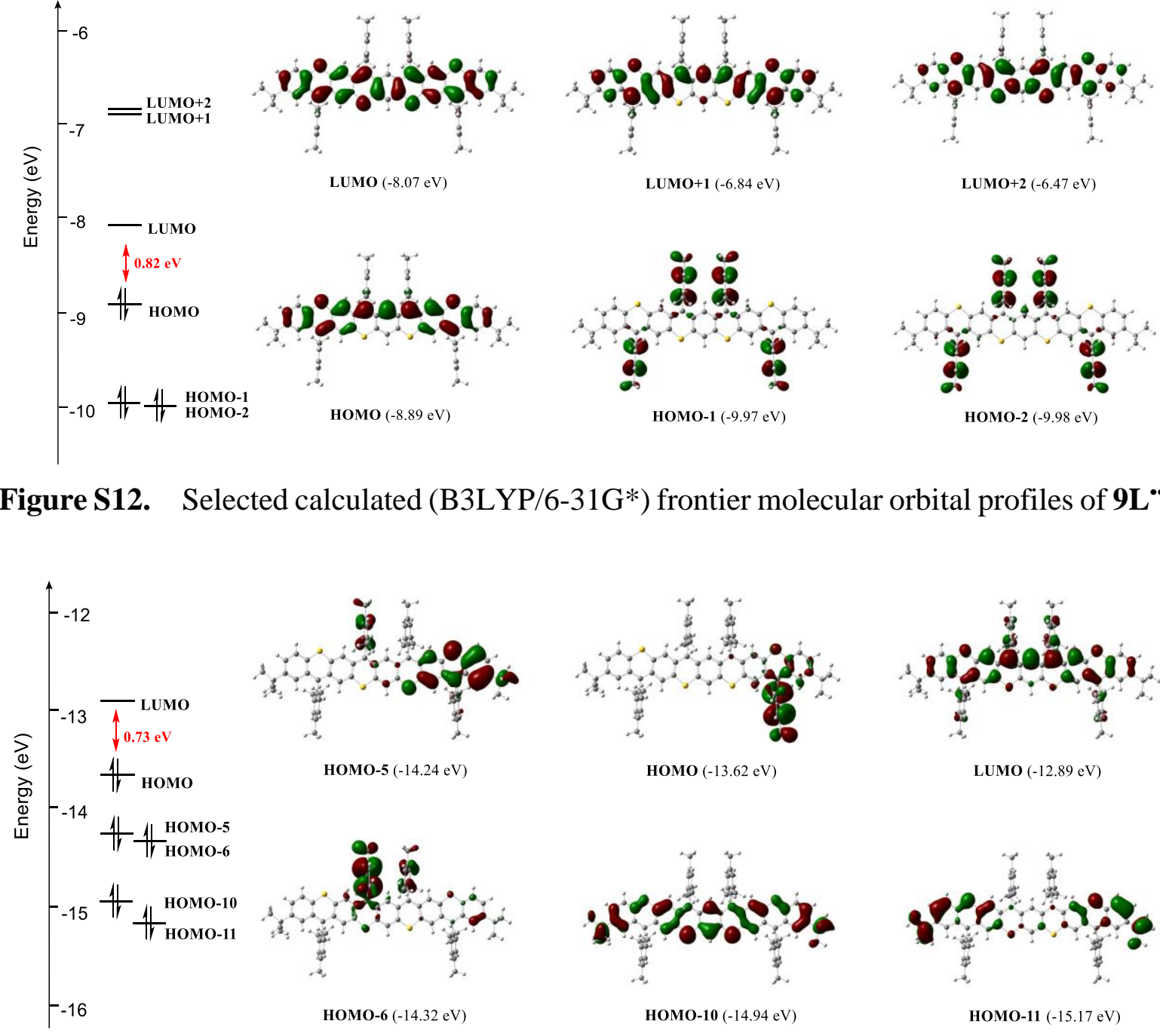

Figure S13. Selected calculated (B3LYP/6-31G*) frontier molecular orbital profiles of $9 \mathbf{L}^{\mathbf{4 +}}$. 
(a)

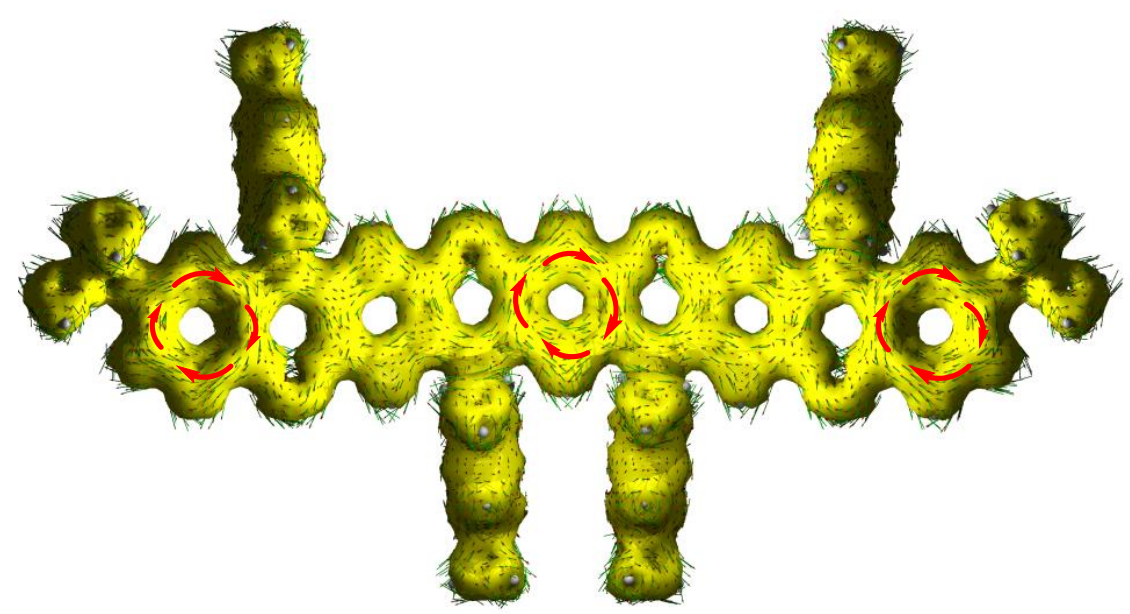

(b)

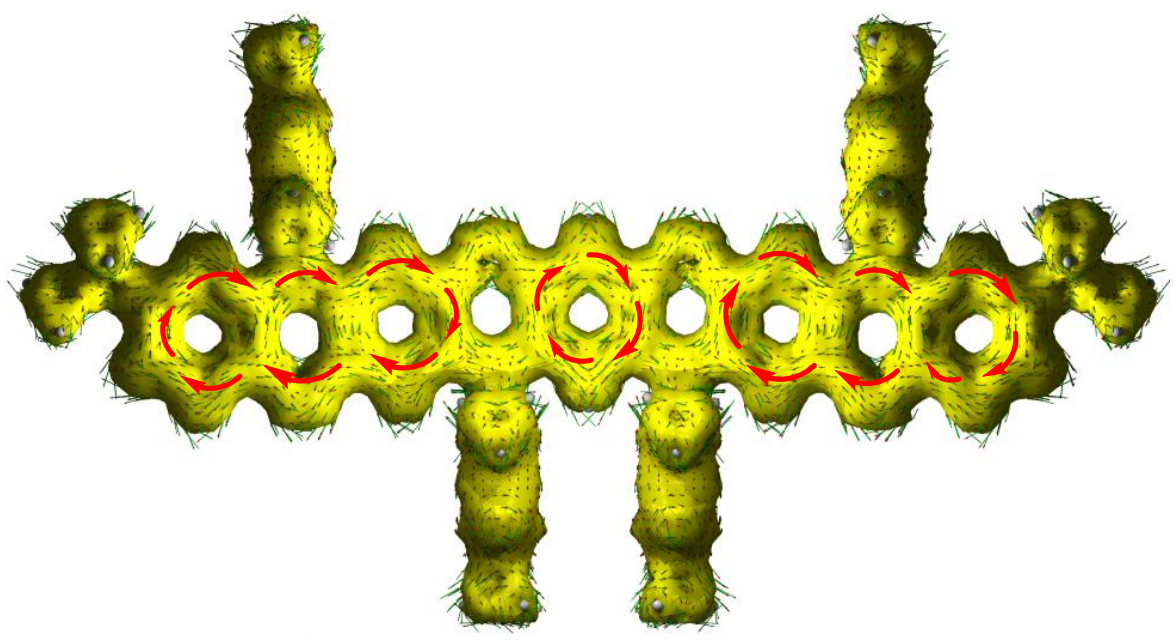

(c)

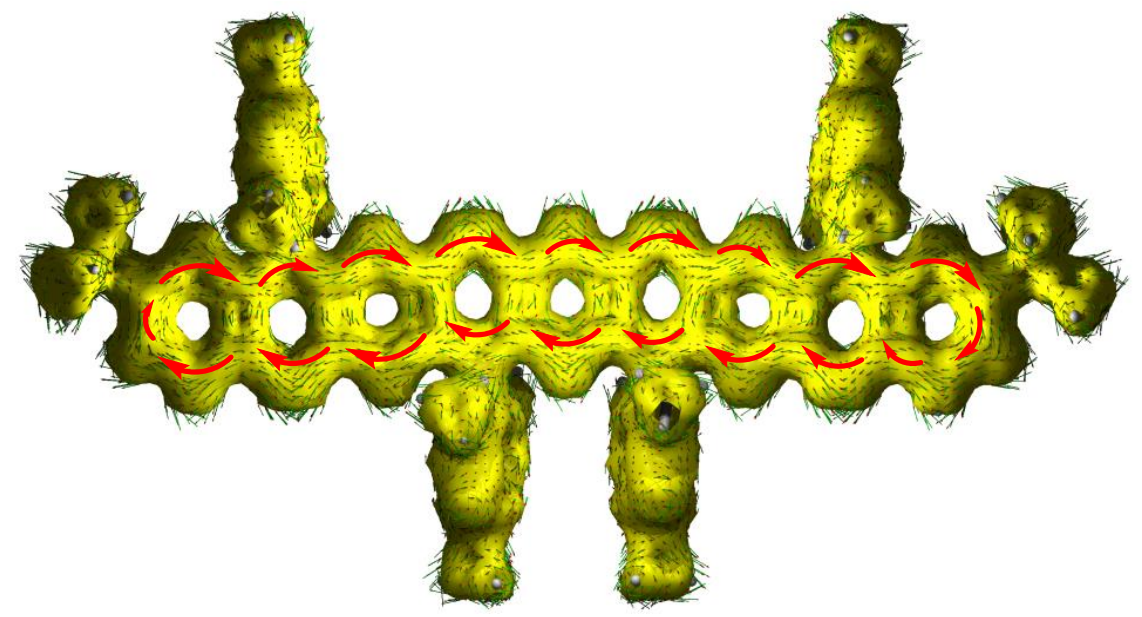

(d)

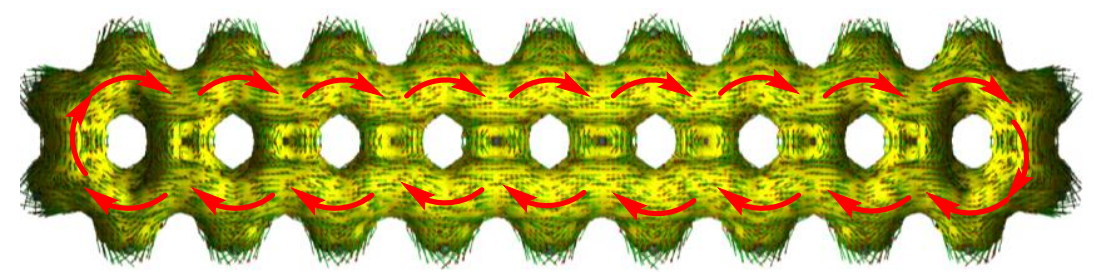

Figure S14. ACID plots of a) $9 \mathbf{L}$, b) $9 \mathbf{L}^{\cdot \cdot 2+}$, c) $9 \mathbf{L}^{4+}$ and d) nonacene at an isosurface value of 0.02. The external magnetic field is applied orthogonal to the plane. 
(a)

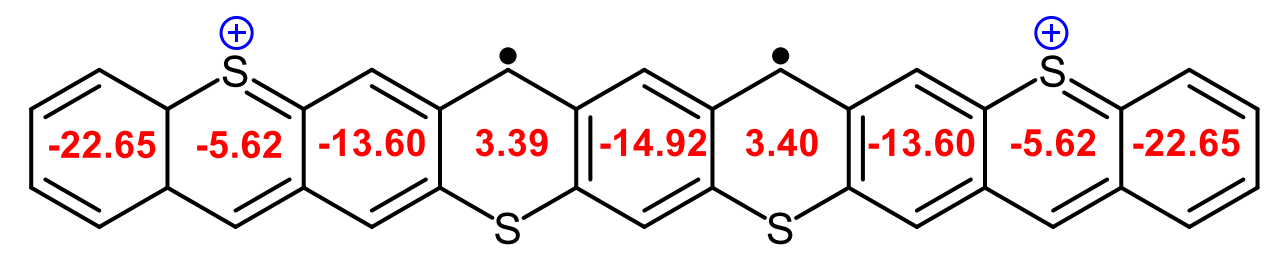

(b)

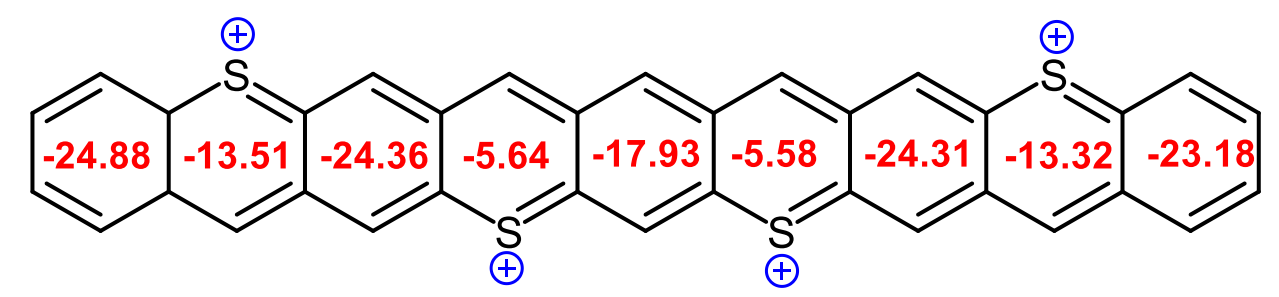

Figure S15. Calculated NICS(1)zz values of (a) $9 \mathbf{L}^{\cdot \cdot 2+}$ and (b) $9 \mathbf{L}^{4+}$.
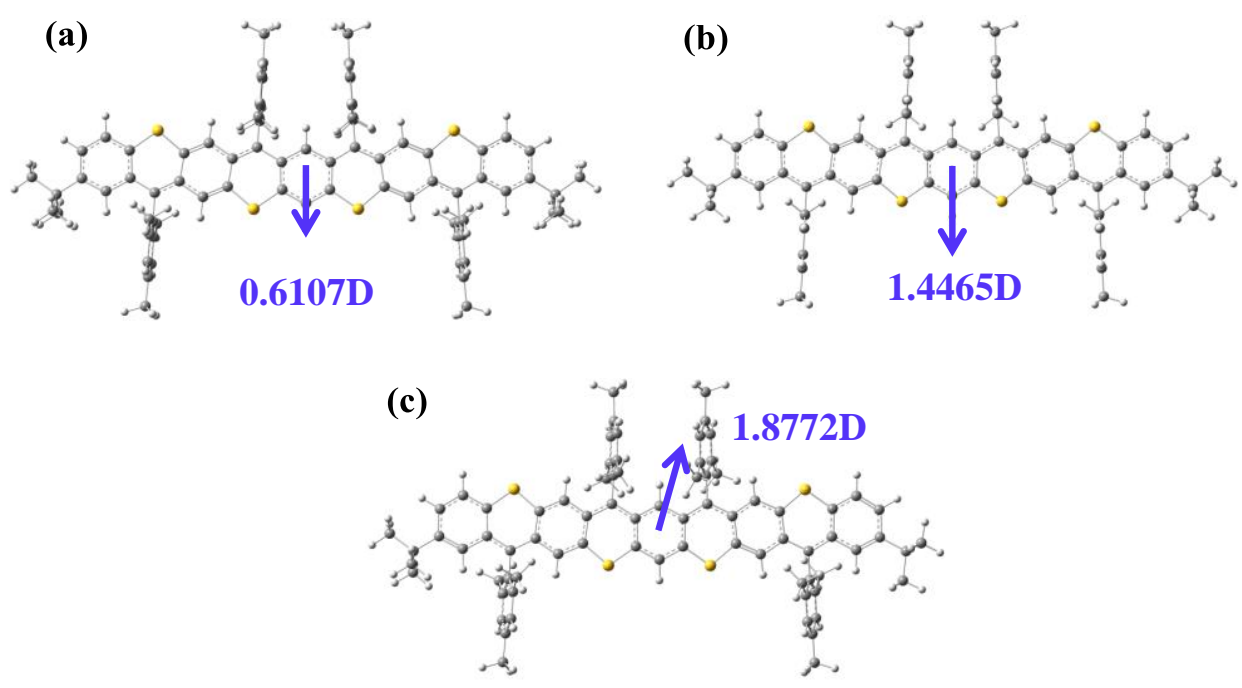

Figure S16. Calculated dipole moments of (a) $9 \mathrm{~L}$, (b) $9 \mathbf{L}^{\cdot{ }^{* 2+}}$ and (c) $9 \mathrm{~L}^{4+}$.

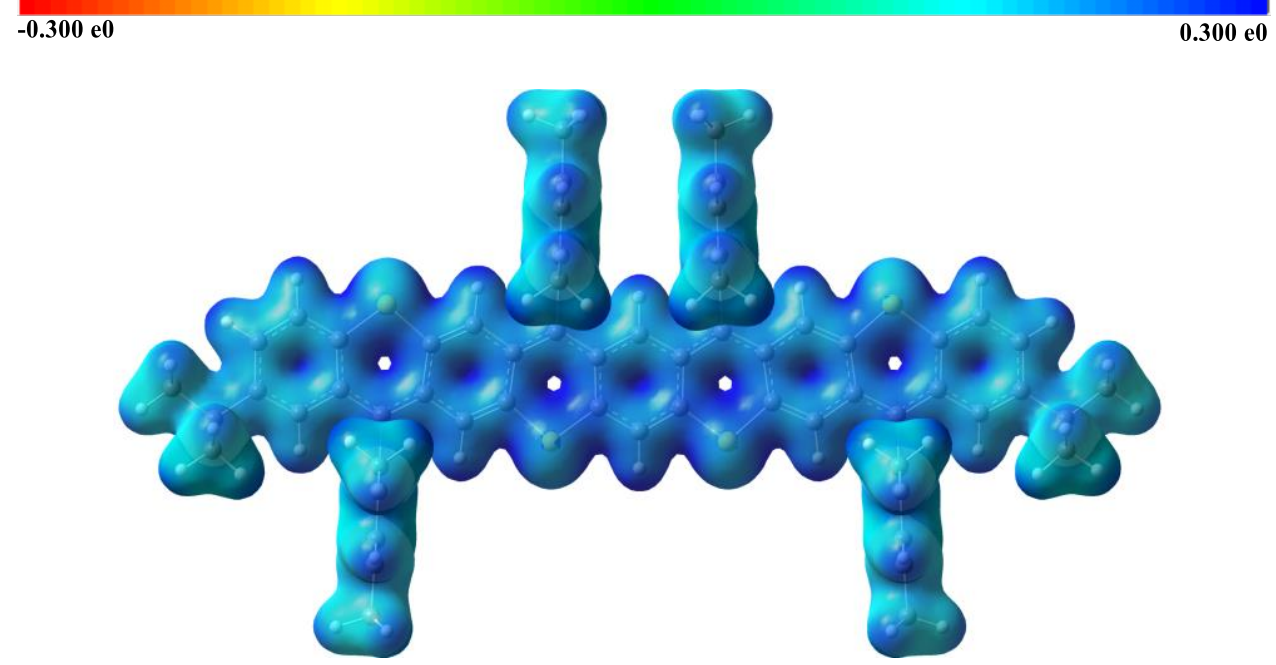

Figure S17. Electrostatic potential maps of $9 \mathbf{L}^{2+}$. 


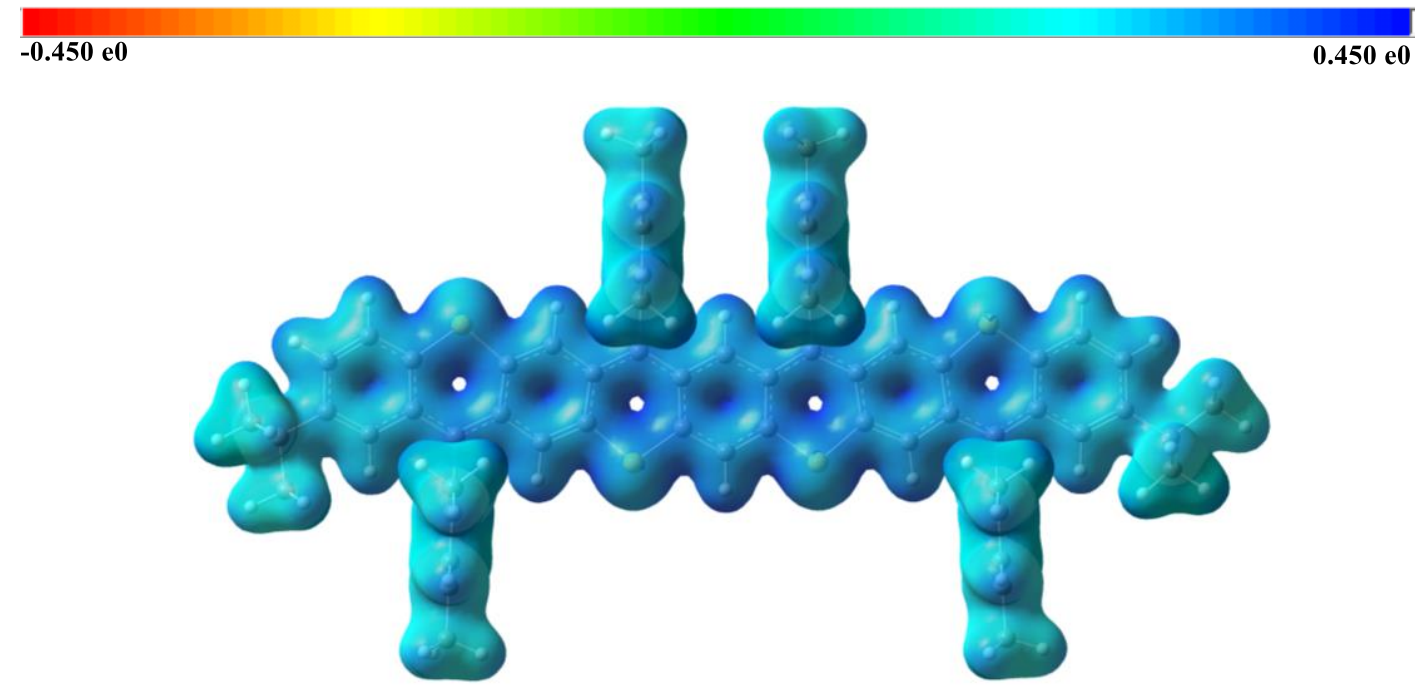

Figure S18. Electrostatic potential maps of $9 \mathbf{L}^{\cdot 3+}$.

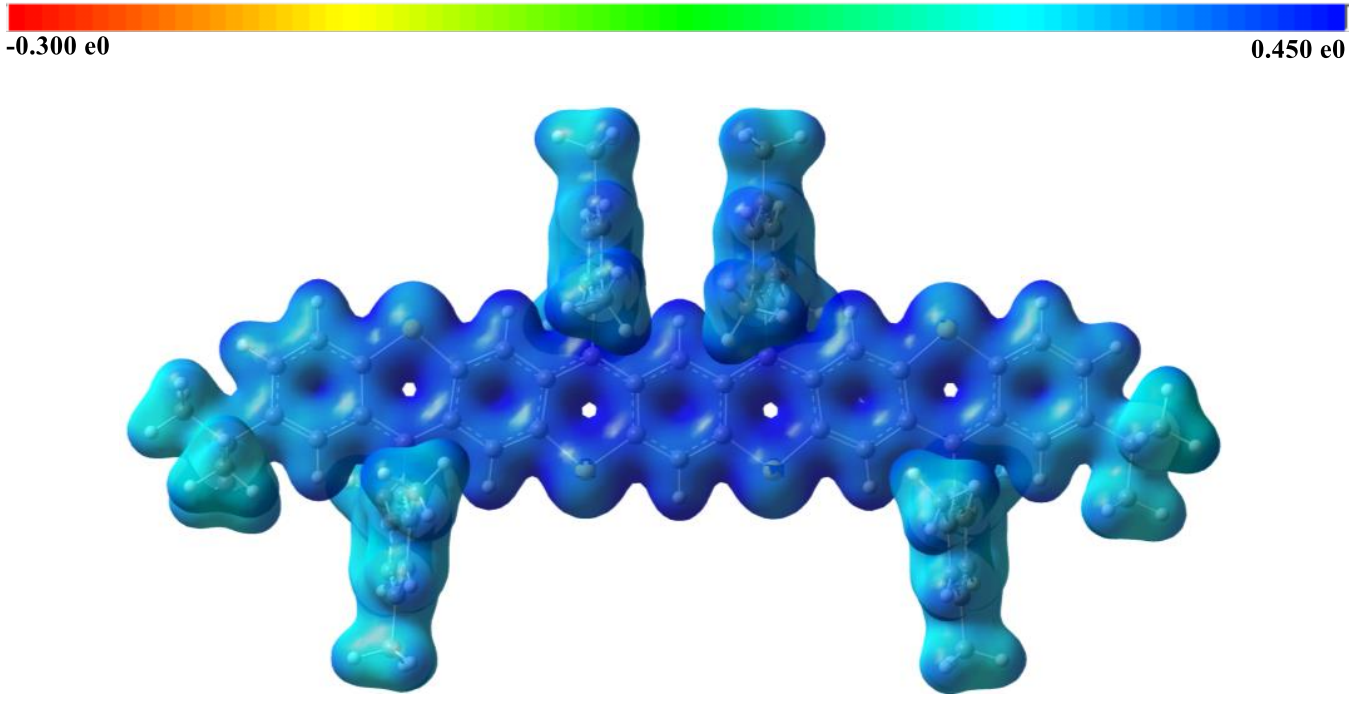

Figure S19. Electrostatic potential maps of $9 \mathbf{L}^{4+}$.

\section{References:}

1. Gaussian 09; Revision A.2; Frisch, M. J.; Trucks, G. W.; Schlegel, H. B.; Scuseria, G. E.; Robb, M. A.; Cheeseman, J. R.; Scalmani, G.; Barone, V.; Mennucci, B.; Petersson, G. A.; Nakatsuji, H.; Caricato, M.; Li, X.; Hratchian, H. P.; Izmaylov, A. F.; Bloino, J.; Zheng, G.; Sonnenberg, J. L.; Hada, M.; Ehara, M.; Toyota, K.; Fukuda, R.; Hasegawa, J.; Ishida, M.; Nakajima, T.; Honda, Y.; Kitao, O.; Nakai, H.; Vreven, T.; Montgomery, J., J. A.; Peralta, J. E.; Ogliaro, F.; Bearpark, M.; Heyd, J. J.; Brothers, E.; Kudin, K. N.; Staroverov, V. N.; Kobayashi, R.; Normand, J.; Raghavachari, K.; Rendell, A.; Burant, J. C.; Iyengar, S. S.; Tomasi, J.; Cossi, M.; Rega, N.; Millam, N. J.; Klene, M.; Knox, J. E.; Cross, J. B.; Bakken, V.; Adamo, C.; Jaramillo, J.; Gomperts, R.; Stratmann, R. E.; Yazyev, O.; Austin, A. J.; Cammi, R.; Pomelli, C.; Ochterski, J. W.; Martin, R. L.; Morokuma, K.; Zakrzewski, V. G.; Voth, G. 
A.; Salvador, P.; Dannenberg, J. J.; Dapprich, S.; Daniels, A. D.; Farkas, Ö.; Foresman, J. B.; Ortiz, J. V.; Cioslowski, J.; Fox, D. J.; Gaussian, Inc., Wallingford CT, 2009.

2. Becke, A. D. J. Chem. Phys. 1993, 98, 5648.

3. Lee, C.; Yang, W.; Parr, R. G. Phys. Rev. B: Condens. Matter 1988, 37, 785.

4. Yanai, T.; Tew, D.; and Handy, N. Chem. Phys. Lett. 2004, 393, 51.

5. Ditchfield, R.; Hehre, W. J.; Pople, J. A. J. Chem. Phys. 1971, 54, 724.

6. Hehre, W. J.; Ditchfield R.; Pople, J. A. J. Chem. Phys. 1972, 56, 2257.

7. Hariharan, P. C.; Pople, J. A. Theor. Chim. Acta 1973, 28, 213.

8. Fallah-Bagher-Shaidaei, H.; Wannere, S. S.; Corminboeuf, C.; Puchta, R.; Schleyer, P. V.

R. Org. Lett. 2006, 8, 863.

9. Geuenich, D.; Hess, K.; Köhler, F.; Herges, R. Chem. Rev. 2005,105, 3758. 


\section{Appendix I: Crystallographic data}

Table S6. Crystal data and structure refinement for 9L.

Empirical formula

Formula weight

Temperature

Wavelength

Crystal system

Space group

Unit cell dimensions

Volume

$\mathrm{Z}$

Density (calculated)

Absorption coefficient

$\mathrm{F}(000)$

Crystal size

Theta range for data collection

Index ranges

Reflections collected

Independent reflections

Completeness to theta $=66.596^{\circ}$

Absorption correction

Max. and min. transmission

Refinement method

Data / restraints / parameters

Goodness-of-fit on $\mathrm{F}^{2}$

Final R indices [I $>2 \operatorname{sigma}(\mathrm{I})]$

$\mathrm{R}$ indices (all data)

Extinction coefficient

Largest diff. peak and hole
$\mathrm{C}_{85} \mathrm{H}_{82} \mathrm{~S}_{4}$

1231.74

100(2) K

$1.54178 \AA$

Triclinic

P-1

$\mathrm{a}=8.0174(4) \AA \quad \alpha=94.788(2)^{\circ}$.

$\mathrm{b}=8.8717(4) \AA \quad \beta=93.499(2)^{\circ}$.

$\mathrm{c}=48.8316(17) \AA \quad \gamma=96.746(2)^{\circ}$.

3428.4(3) $\AA^{3}$

2

$1.193 \mathrm{Mg} / \mathrm{m}^{3}$

$1.609 \mathrm{~mm}^{-1}$

1312

$0.326 \times 0.236 \times 0.092 \mathrm{~mm}^{3}$

2.731 to $66.596^{\circ}$.

$-9<=\mathrm{h}<=9,-10<=\mathrm{k}<=10,-57<=\mathrm{l}<=58$

35985

$11947[\mathrm{R}($ int $)=0.0949]$

$98.5 \%$

Semi-empirical from equivalents

0.7528 and 0.5984

Full-matrix least-squares on $\mathrm{F}^{2}$

11947 / 661 / 1030

1.020

$\mathrm{R} 1=0.0937, \mathrm{wR} 2=0.2230$

$\mathrm{R} 1=0.1518, \mathrm{wR} 2=0.2566$

$\mathrm{n} / \mathrm{a}$

0.780 and -0.566 e. $\AA^{-3}$

Note: The crystal is triclinic, space group P-1. The asymmetric unit contains one molecule of the compound $\mathrm{C}_{78} \mathrm{H}_{74} \mathrm{~S}_{4}$ and one toluene. The two ends of the molecules were disordered into two positions with occupancy ratio $=50: 50$. The ratio has to be 50:50 because one of the disordered parts clashed with the adjacent molecule. One of the $\mathrm{S}$ atom was also disordered into two positions with occupancy ratio $=50: 50$. The toluene molecule is also disordered into two positions (Occupancy ratio+50:50). Restraints in bond lengths and thermal parameters were applied to the disordered atoms. Final R values are R1=0.0937 and wR2 $=0.2566$ for 2-theta up to $133^{\circ}$. 
Table S7. Crystal data and structure refinement for $9 \mathbf{L}^{\cdot 3+}$.

Empirical formula

note below)

Formula weight

Temperature

Wavelength

Crystal system

Space group

Unit cell dimensions

Volume

Z

Density (calculated)

Absorption coefficient

$\mathrm{F}(000)$

Crystal size

Theta range for data collection

Index ranges

Reflections collected

Independent reflections

Completeness to theta $=25.242^{\circ}$

Absorption correction

Max. and min. transmission

Refinement method

Data / restraints / parameters

Goodness-of-fit on $\mathrm{F}^{2}$

Final R indices [I $>2 \operatorname{sigma}(\mathrm{I})]$

$\mathrm{R}$ indices (all data)

Extinction coefficient

Largest diff. peak and hole
$\mathrm{C}_{84} \mathrm{H}_{87} \mathrm{Cl}_{6} \mathrm{~F}_{18} \mathrm{~S}_{4} \mathrm{Sb}_{3}\left(\mathbf{9 L}^{\cdot 3+}\right.$ and solvents, see

2144.72

100(2) K

$0.71073 \AA$

Triclinic

P-1

$\mathrm{a}=12.2451(5) \AA \quad \alpha=107.5600(10)^{\circ}$.

$\mathrm{b}=16.1940(6) \AA \quad \beta=92.3800(10)^{\circ}$.

$\mathrm{c}=24.0023(9) \AA \quad \gamma=95.341(2)^{\circ}$.

4505.9(3) $\AA^{3}$

2

$1.581 \mathrm{Mg} / \mathrm{m}^{3}$

$1.243 \mathrm{~mm}^{-1}$

2144

$0.359 \times 0.199 \times 0.112 \mathrm{~mm}^{3}$

2.249 to $28.283^{\circ}$.

$-16<=\mathrm{h}<=16,-21<=\mathrm{k}<=21,-31<=\mathrm{l}<=31$

118622

$22349[\mathrm{R}($ int $)=0.0406]$

$99.9 \%$

Semi-empirical from equivalents

0.7459 and 0.6492

Full-matrix least-squares on $\mathrm{F}^{2}$

22349 / 247 / 1151

1.034

$\mathrm{R} 1=0.0502, \mathrm{wR} 2=0.1374$

$\mathrm{R} 1=0.0668, \mathrm{wR} 2=0.1490$

$\mathrm{n} / \mathrm{a}$

2.722 and -1.642 e. $\AA^{-3}$

Note: The crystal is triclinic, space group $\mathrm{P}-1$. The asymmetric unit contains one cation of $\mathrm{C}_{78} \mathrm{H}_{74} \mathrm{~S}_{4}\left(\mathbf{9 L}^{\cdot 3+}\right.$ ), four anions $\mathrm{SbF}_{6}$ (two of them have half occupancy), three $\mathrm{CH}_{2} \mathrm{Cl}_{2}$ and half of a hexane. One of the $\mathrm{SbF}_{6}$ and the hexane were disordered into two positions with occupancy ratios $=60: 30$ and 70:30 respectively. Restraints in bond lengths and thermal parameters were applied to the disordered atoms. Final R values are R1 $=0.0502$ and $w R 2=0.1490$ for 2-theta up to $56^{\circ}$. 
5. Appendix II: ${ }^{1} \mathrm{H}$ NMR and ${ }^{13} \mathrm{C}$ NMR spectra of all new compounds

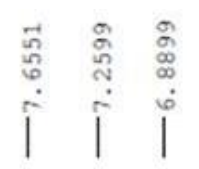
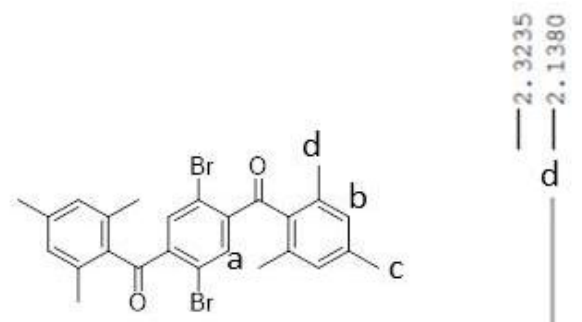

d

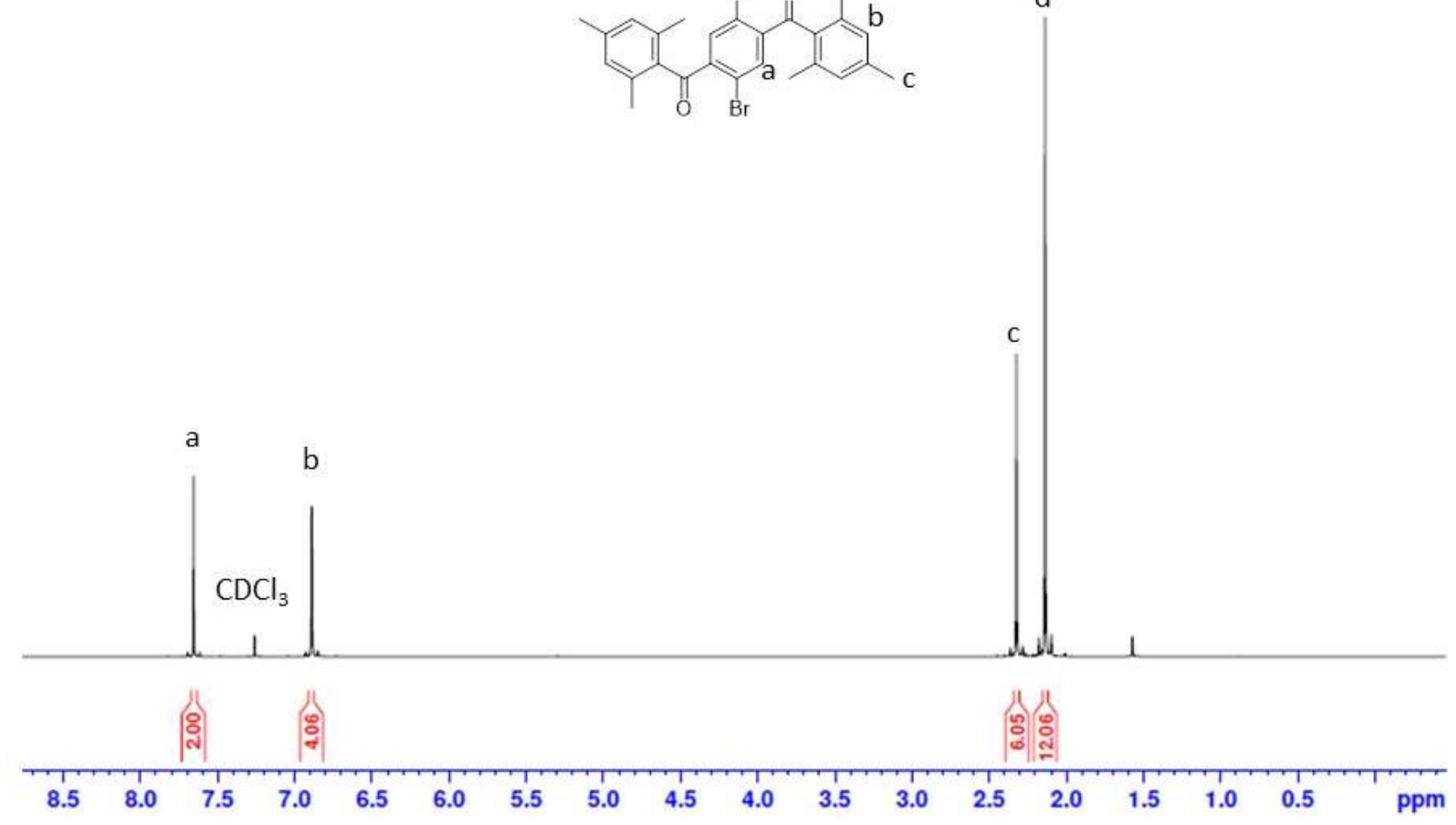

Figure S20. ${ }^{1} \mathrm{H}$ NMR spectrum of $1\left(500 \mathrm{MHz}, \mathrm{CDCl}_{3}\right)$. 


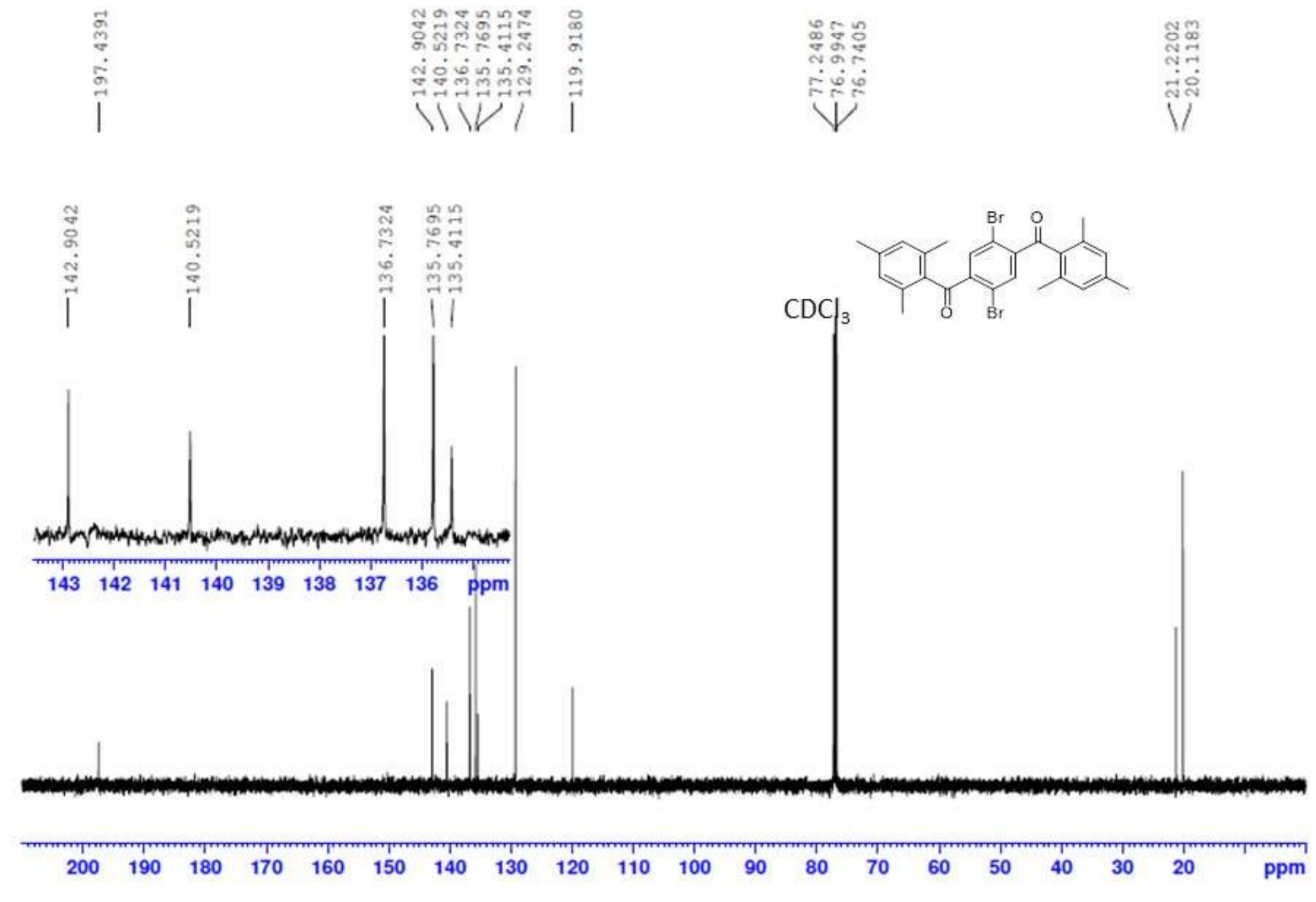

Figure S21. ${ }^{13} \mathrm{C}$ NMR spectrum of $1\left(125 \mathrm{MHz}, \mathrm{CDCl}_{3}\right)$. 

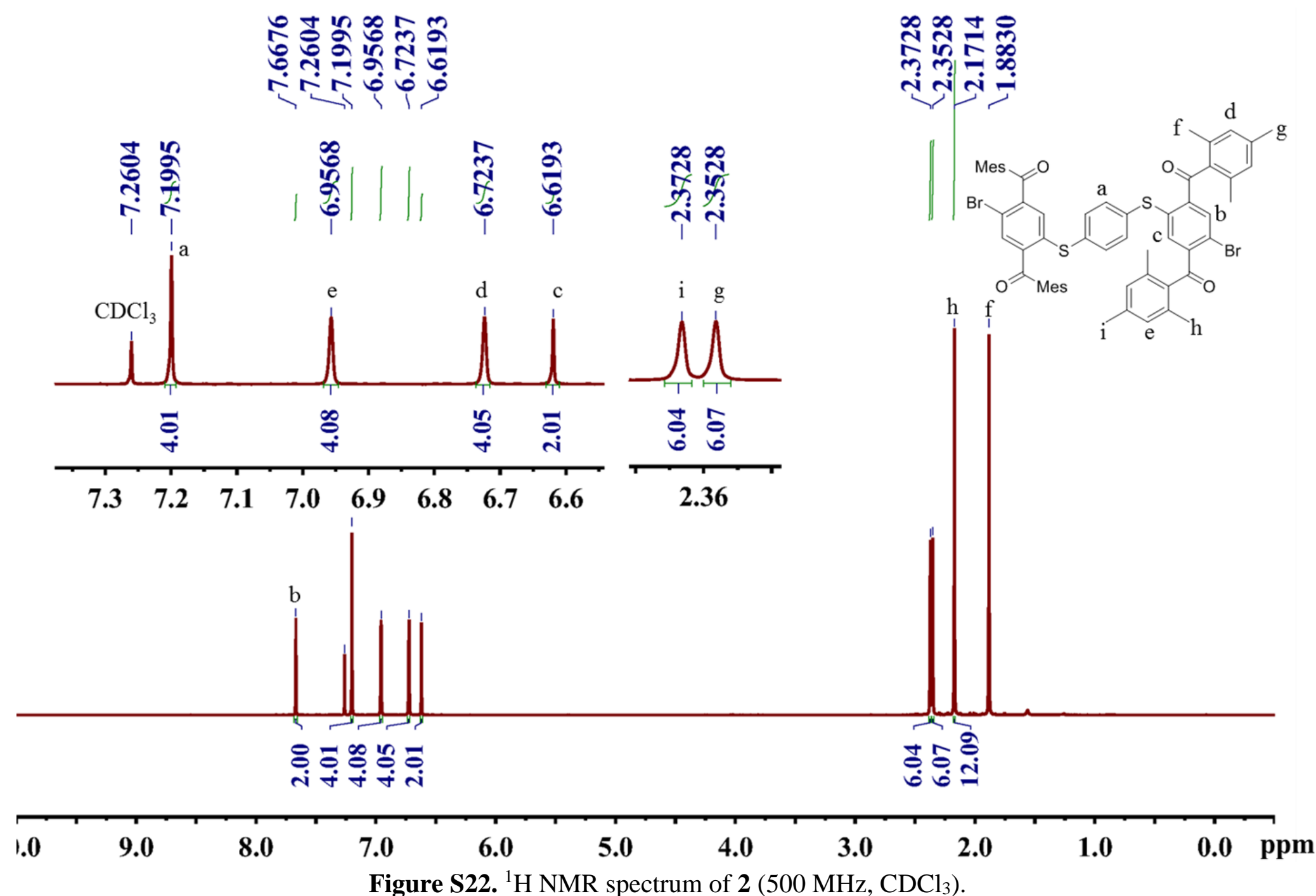


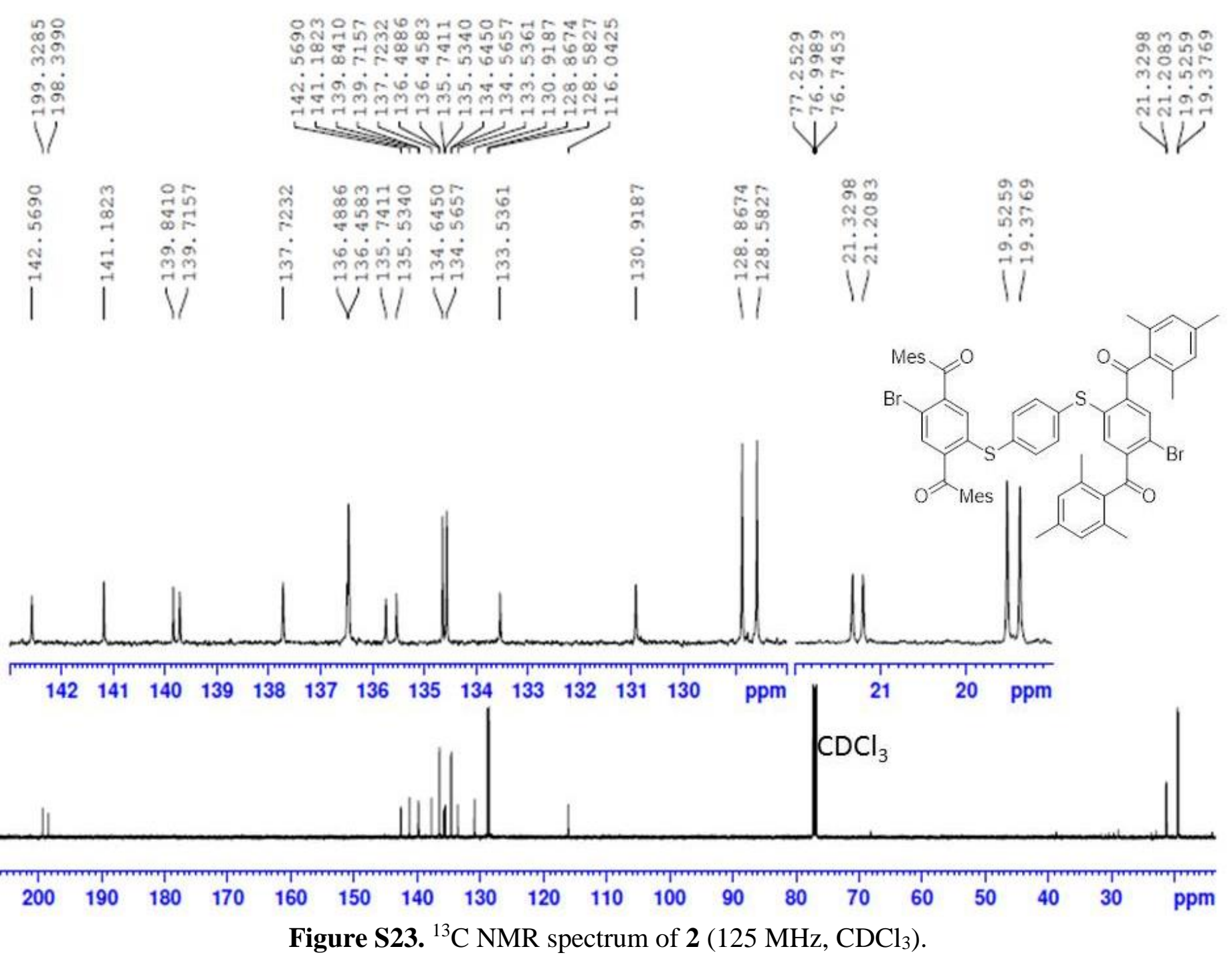




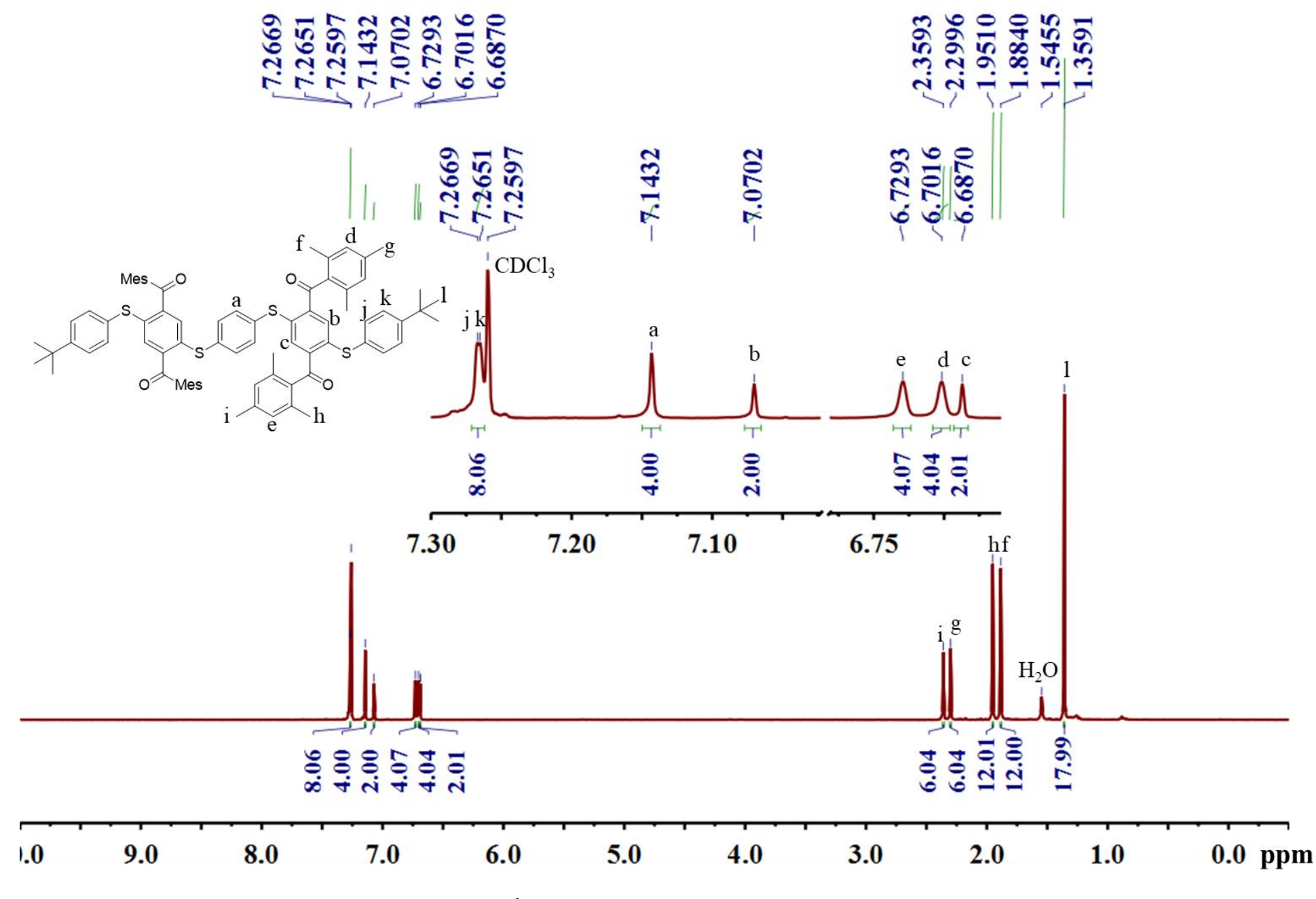

Figure S24. ${ }^{1} \mathrm{H}$ NMR spectrum of $\mathbf{3}\left(500 \mathrm{MHz}, \mathrm{CDCl}_{3}\right)$. 


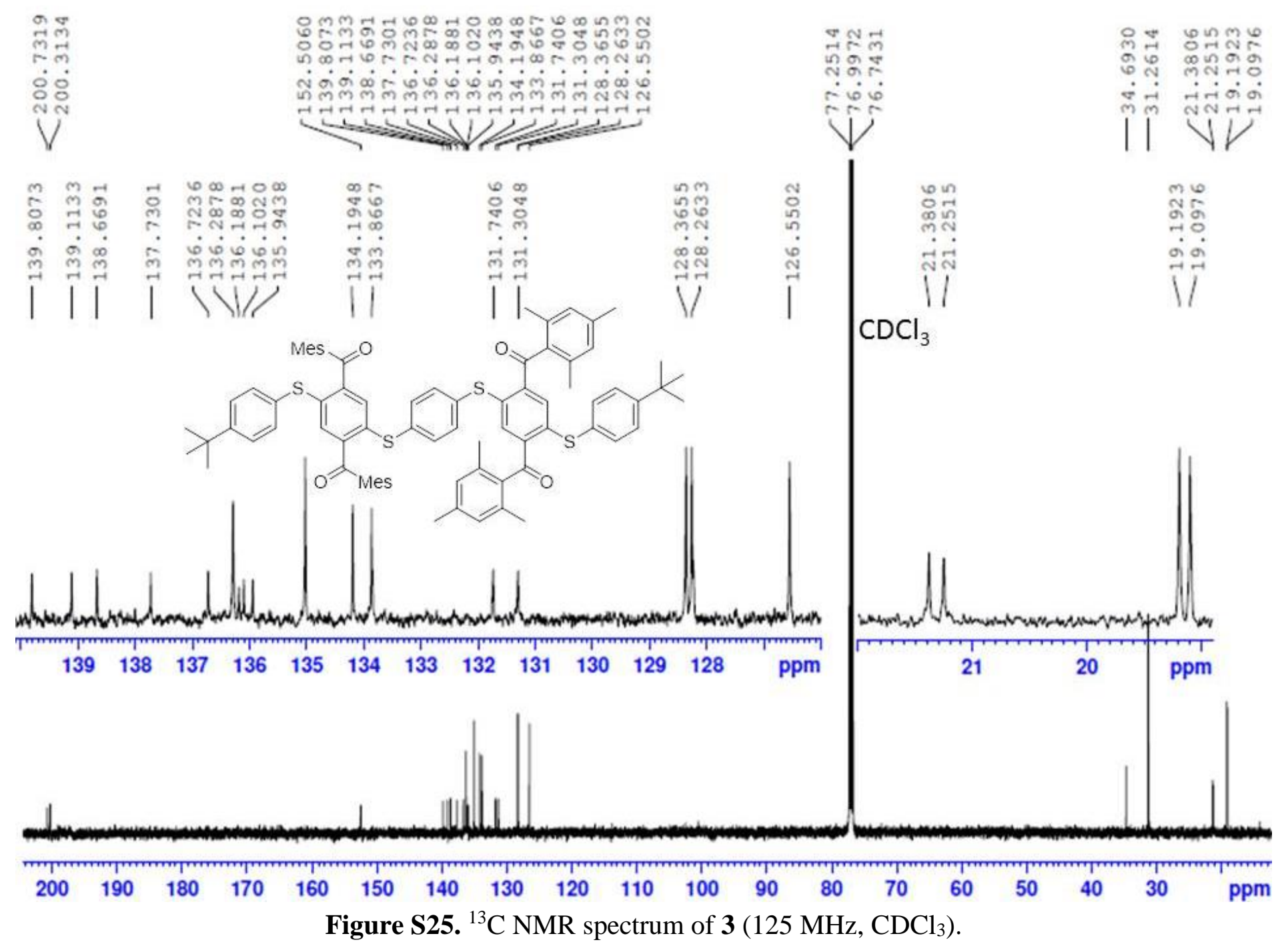




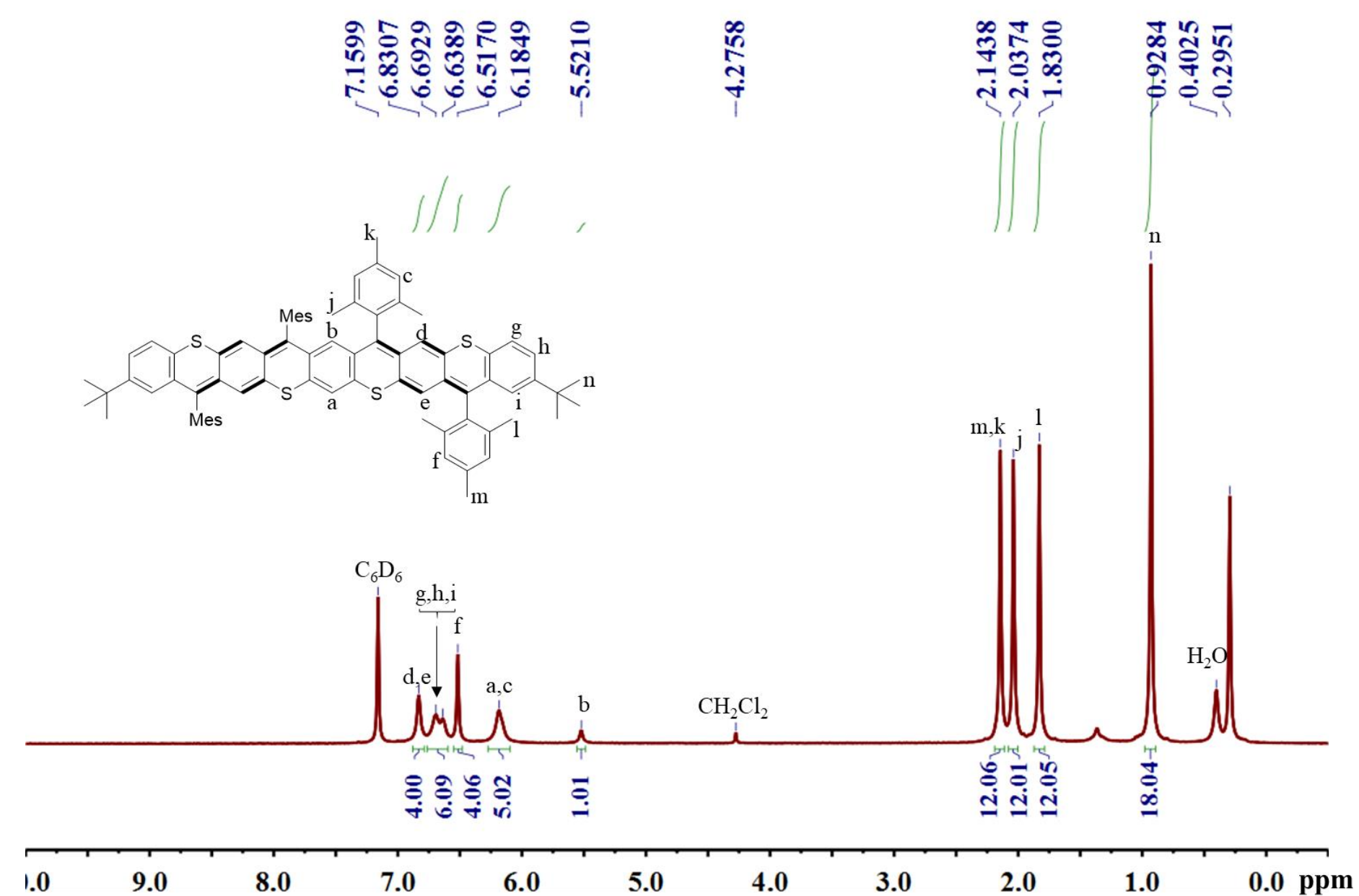

Figure S26. ${ }^{1} \mathrm{H}$ NMR spectrum of $\mathbf{9 L}\left(500 \mathrm{MHz}, \mathrm{C}_{6} \mathrm{D}_{6}\right)$. The aromatic region was significantly broaded in chlorinated solvents (such as $\mathrm{CDCl}_{2} \mathrm{CDCl}_{2}$ ) presumably due to aggregation. 


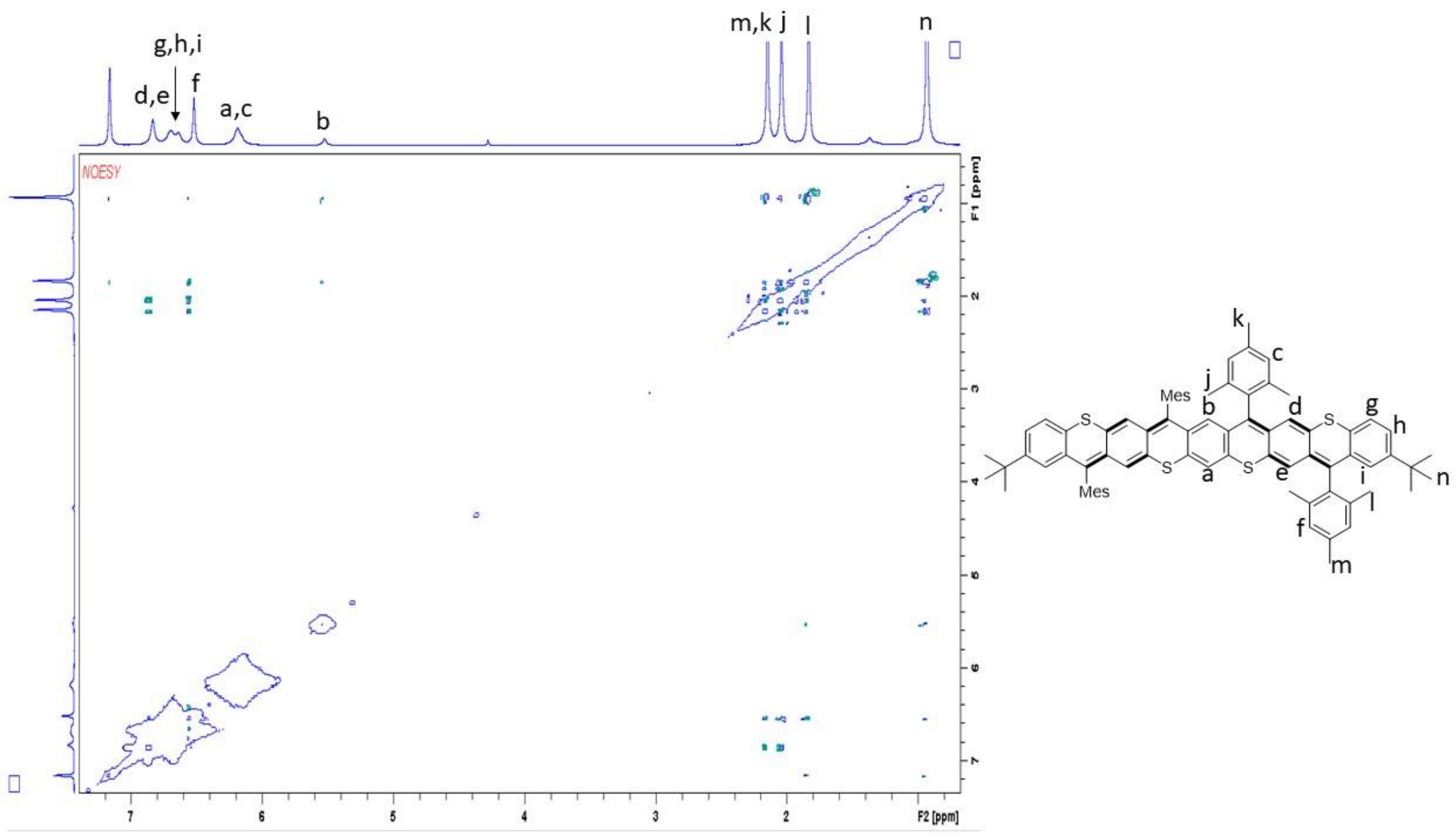

Figure S27. 2D NOESY NMR spectrum of 9L with assignments $\left(500 \mathrm{MHz}, d_{6}\right.$-benzene, $\left.25^{\circ} \mathrm{C}\right)$. 


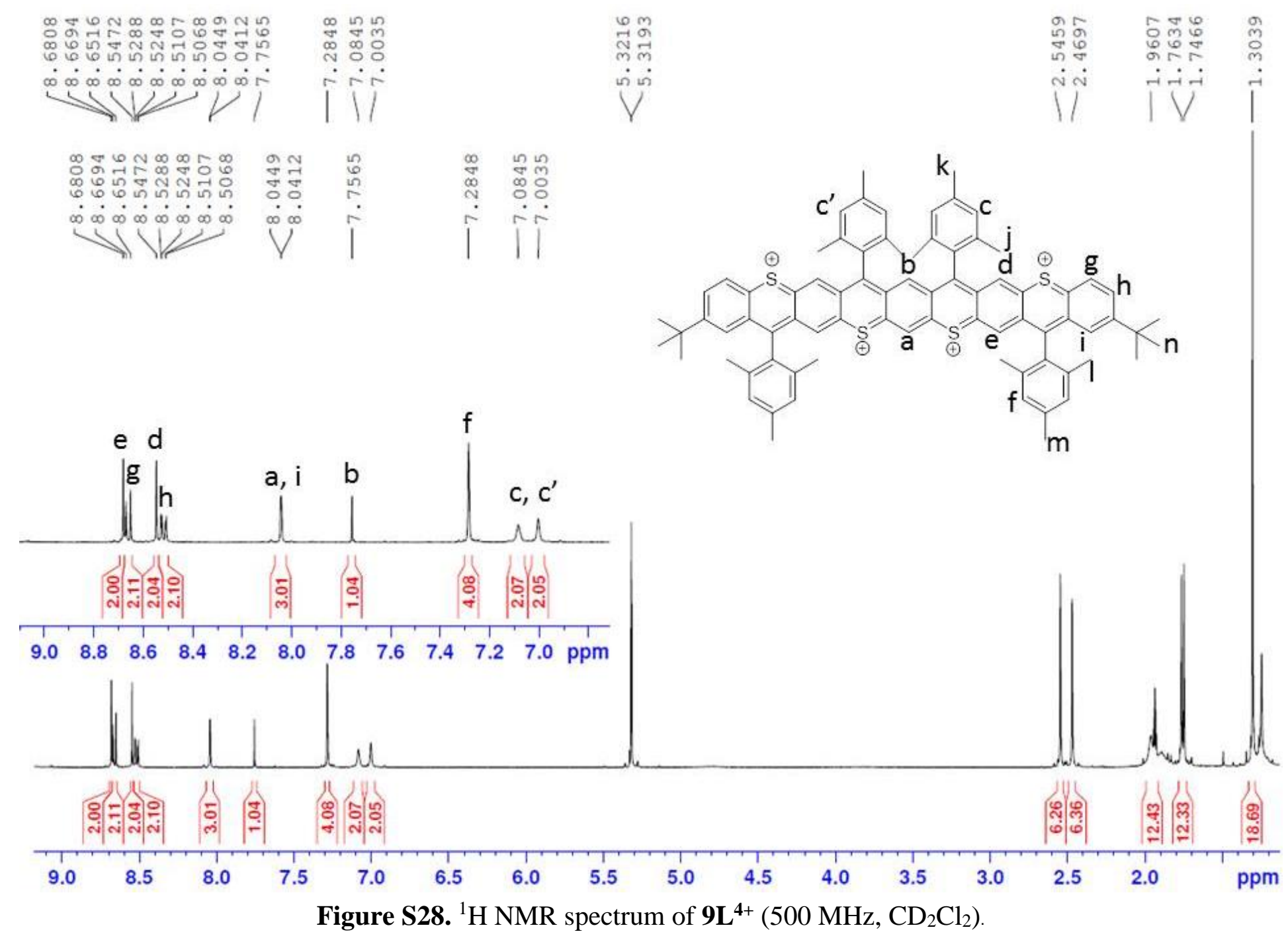




\section{Appendix III: High Resolution mass spectrum of 9L}

Analysis Info

Analysis Name

Method

Sample Name

Comment
Acquisition Date $\quad$ 12/30/2016 12:52:15 PM

Operator default user

Instrument / Ser\# micrOTOF-Q II 10269

Acquisition Parameter

Scan Begin

DatalChemistryl2016 Samplel201612l1230l9L d 